\title{
An Embedded Statistical Method for Coupling Molecular Dynamics and Finite Element Analyses
}

\author{
E. Saether ${ }^{1 *}$, V. Yamakov ${ }^{2}$, and E.H. Glaessgen ${ }^{1}$

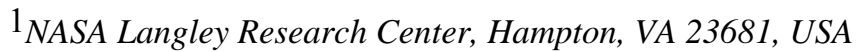 \\ ${ }^{2}$ National Institute of Aerospace, Hampton, VA 23666, USA
}

\begin{abstract}
SUMMARY
The coupling of molecular dynamics (MD) simulations with finite element methods (FEM) yields computationally efficient models that link fundamental material processes at the atomistic level with continuum field responses at higher length scales. The theoretical challenge involves developing a seamless connection along an interface between two inherently different simulation frameworks. Various specialized methods have been developed to solve particular classes of problems. Many of these methods link the kinematics of individual MD atoms with FEM nodes at their common interface, necessarily requiring that the finite element mesh be refined to atomic resolution. Some of these coupling approaches also require simulations to be carried out at $0 \mathrm{~K}$ and restrict modeling to two-dimensional material domains due to difficulties in simulating full threedimensional material processes. In the present work, a new approach to MD-FEM coupling is developed based on a restatement of the standard boundary value problem used to define a coupled domain. The method replaces a direct linkage of individual MD atoms and finite element (FE) nodes with a statistical averaging of atomistic displacements in local atomic volumes associated with each FE node in an interface region. The FEM and MD computational systems are effectively independent and communicate only through an iterative update of their boundary conditions. With the use of statistical averages of the atomistic quantities to couple the two computational schemes, the developed approach is referred to as an embedded statistical coupling method (ESCM). ESCM provides an enhanced coupling methodology that is inherently applicable to three-dimensional domains, avoids discretization of the continuum model to atomic scale resolution, and permits finite temperature states to be applied.
\end{abstract}

\footnotetext{
${ }^{*}$ Corresponding author: Tel.: +757-864-8079; fax: +757-864-8911.

E-mail address: e.saether@nasa.gov (E. Saether)
} 


\section{INTRODUCTION}

The emerging field of nanomechanics is providing a new focus in the study of the mechanics of materials, particularly that of simulating fundamental atomic mechanisms involved in the initiation and evolution of damage. These simulations are commonly based on either quantum mechanics (ab-initio, tight-binding (TB) or density-functional theory (DFT)) methods, on classical molecular dynamics (MD), or molecular statics (MS) methods. These predictions of material behavior at nanometer length scales promise the development of physics-based 'bottom-up' multiscale analyses that can aid in understanding the evolution of failure mechanisms across length scales. However, modeling atomistic processes quickly becomes computationally intractable as the system size increases. With current computer technology, the computational demands of modeling suitable domain sizes (on the order of hundreds of atoms for quantum mechanics-based methods, and potentially billions of atoms for classical mechanicsbased methods) and integrating the governing equations of state over sufficiently long time intervals, quickly reaches an upper bound for practical analyses. In contrast, continuum mechanics methods such as the finite element method (FEM) provide an economical numerical representation of material behavior at length scales in which continuum assumptions apply. However, all constitutive relationships, kinematics, etc, must be assumed a priori.

The concept of bridging length scales by concurrently coupling atomistic and continuum computational paradigms is particularly attractive as a highly efficient means of reducing the computational cost of simulations in cases that require modeling of relatively large material domains to capture the complete deformation field, but where atomic and subatomic refinement is needed only in very localized regions. Such computational issues arise in modeling crack nucleation and propagation, and in modeling dislocation formation and interaction. By using coupled models, the size limitations of the atomistic simulation can be minimized by embedding an inner region where complex dynamic processes and large deformation gradients exist within an outer domain where the deformation gradients are small so that a continuum finite element method (FEM) representation of the material becomes appropriate. 
Over the past decade, various methods have been developed to address different problems involving atomistically large material domains [1-12]. The most challenging problem in developing these coupled methods is the formulation of a seamless computational connection along an interface between different material representations. A brief review of several representative coupling procedures follows to illustrate the current state-of-the-art.

In coupling atomistic and continuum material representations, the continuity of material properties must be maintained while transitioning from individual atoms interacting through nonlocal forces to the local stress-strain field formalism of continuum mechanics. For crack problems, the early efforts of Gumbsch and Beltz [10] led to the development of the Finite Element - Atomistic (FEAt) coupling procedure that combined an embedded MD system with a finite element domain. A generalized formulation of conventional FEM, which allows FEM nodes to be considered as coarse-grained MD "atoms" led to another computational scheme for atomistic-continuum coupling called Coarse Grained Molecular Dynamics (CGMD). A detailed discussion of CGMD is given by Rudd and Broughton [11,12]. In yet another coupling method, the Coupling of Length Scales (CLS) method [2], the nodes in a finite element model representing the continuum region are directly connected to the atoms in an atomistic region forming an interface of "pad" atoms. The region of "pad" atoms, used in this and other atomistic-continuum coupling methods, serves to minimize surface tension effects on the atoms in the atomistic system but also introduces a constraint due to the elasticity of the interface region. The constraining effect of this region is generally considered insignificant and is ignored.

The Quasicontinuum (QC) method, reviewed by Miller and Tadmor [6], is formally based on an entirely atomistic description of the material domain. However, for computational efficiency, regions are identified in which discrete atoms may be grouped to form a local continuum. The particular representation used is determined by evaluating the magnitude of local deformation gradients and dictates the treatment of "representative atoms" or "repatoms." In the QC formalism, "non-local repatoms" are used to represent "real" atoms to form atomistic regions treated by MS/MD methods while "local repatoms" are used to define continuum domains by applying both the 
Cauchy-Born rule [13] and aspects of FEM. The interaction of local and nonlocal repatoms at the atomistic/continuum interface leads to the generation of "ghost forces" that must be mitigated through the introduction of "dead loads" that are iterated for selfconsistency in the force balance at the interface between subregions.

Another representative coupling approach is the bridging method of Xiao and Belytschko [7] and is based on an overlay approach in which MD and FEM representations are superposed in an interface region. This method allows interpolated FEM nodal displacements to be associated with atomic displacements in the bridging domain.

The Coupled Atomistic/Dislocation Dynamics (CADD) method of Shilkrot et al. [8] is specifically designed to simulate, identify and pass dislocations between atomistic and continuum domains. The method was originally limited to $0 \mathrm{~K}$ simulations [8]; but has been recently extended to include finite temperature effects in the MD system by linking the MD to a quasistatic FEM domain through a thermal damping region [9]. Currently, CADD uses a two-dimensional material representation due to the complexity of passing fully three-dimensional dislocations between the MD and FEM domains.

A common feature of many of these approaches [1-12] for coupling atomistic and continuum representations is the refinement of the finite element (FE) mesh to atomic length scales to link the kinematics of the FE nodes to that of the discrete atoms along an interface. In this paper, approaches that relate atoms and FE nodes in a one-to-one manner, or through a form of interpolation, will be referred to as direct coupling (DC) approaches.

While DC approaches are straightforward, the fundamental difficulty in their development lies in the inherent differences between the atomistic and continuum computational models. The physical state of the atomistic region is described through nonlocal interatomic forces between discrete atoms of given position and momentum, while the physical state of the continuum region is described through continuous stressstrain fields that reflect local statistical averages of atomic interactions at larger length and time scales. In general, the formal connection between continuum and discrete quantities can only be achieved through an adequate statistical averaging over scales where the discreteness of the atomic structure can be approximated as a continuum. A 
consequence of some DC interfacing strategies in their initial formulation, such as QC, required that the analysis be performed quasistatically at $0 \mathrm{~K}$. Further details of the direct coupling methods may be found in the original publications and in several general review papers [4-6].

In this paper, an alternative approach to the $\mathrm{DC}$ approaches is proposed to construct a coupled MD-FEM system. The approach is based on solving a coupled boundary value problem (BVP) at the MD/FE interface for a $\mathrm{MD}$ region embedded within a FEM domain. The method uses statistical averaging over both time and volume in atomistic subdomains at the $\mathrm{MD} / \mathrm{FE}$ interface to determine nodal displacement boundary conditions for the continuum FE model. These enforced displacements, in turn, generate interface reaction forces that are applied as constant traction boundary conditions [14-16] between updates of the FEM solution to the atoms within the localized MD subdomains. Thus, the present approach may be described as a local-nonlocal BVP because it relates local continuum nodal quantities with nonlocal statistical averages of atomistic quantities over selected atomic subdomains. An iterative procedure between the MD statistical displacements and the FEM reaction forces ensures continuity at the interface. In this way, the problem of redefining continuum variables at the atomic scale is avoided, and the developed interface approach links different time and length scales between the MD region and FEM domain.

With the emphasis of using statistical averages to couple the two computational schemes, the developed approach is identified as a statistical coupling (SC) approach. Based on the SC approach, the developed MD-FEM coupling method is referred to as the embedded statistical coupling method (ESCM). ESCM provides an enhanced coupling methodology that is inherently applicable to three-dimensional domains, avoids discretization of the continuum model to atomic scale resolution, permits arbitrary temperatures to be applied, and treats, in a rigorous manner, the compensation of surface effects in the MD system.

This paper will detail the ESCM approach for coupling MD and FEM computational domains for the case of systems that reach thermodynamic equilibrium or evolve quasistatically. While there is no principle difficulty in implementing this approach for 
non-equilibrium systems, it is beneficial to first consider the case of equilibrium simulations to illustrate initial applications of this methodology.

The remainder of this paper is organized as follows. Section 2 describes the structure of the coupled MD-FEM model. This includes discussions of the MD and FEM material representations, the coupling interface, and the iterative MD-FEM coupling methodology. Section 3 presents several validation studies to substantiate the accuracy of the developed methodology. Section 4 presents concluding remarks on the overall effectiveness of the ESCM. Details of internal force calculations involved in the coupling procedure and a discussion of model generation are contained in separate appendices.

\section{THE ESCM MODEL}

The ESCM approach is developed to reduce computational costs incurred while simulating "large" volumes of material by embedding an inner atomistic MD system within a surrounding continuum FEM domain. In principal, the shape of the atomistic region may be arbitrary as shown in Figure 1; however, for simplicity, the special case of a circular region is utilized in the present work. Similarly, although any constitutive behavior may be assumed for the FEM domain, the present study considers a linear elastic continuum.

The structure of the ESCM model consists of four regions: 1) an Inner MD Region; 2) an Interface MD Region wherein MD and finite elements are superimposed; 3) a Surface MD Region that does not interact with the FE nodes but is used to compensate for atomic free edge effects; and 4) a FEM domain in which standard finite element equations apply. These four regions are depicted in Figure 2.

Complete details of the ESCM procedure will be presented by discussing general aspects of the MD and FEM computational systems, followed by specific details of the $\mathrm{MD} / \mathrm{FE}$ Interface, the Surface MD Region and the MD-FEM coupling procedure.

\subsection{MD and FEM model components}


The Inner MD Region is used to model material phenomena at the atomistic level. This Inner MD Region should be large enough to ensure a statistically smooth transition from a continuum to an atomistic representation while modeling any of the types of processes (e.g., dislocation formation, void nucleation, or crack propagation) that are required by the simulation. Together, the Inner, Interface, and Surface MD Regions constitute the complete MD system.

It is important to emphasize that the partitioning of the MD system into different regions is not a physical separation of the system. An atom assigned to a particular location freely interacts with atoms in its interaction neighborhood that may reside in a different region. Thus, the overall simulation is performed using any conventional MD technique without any imposition of direct kinematic constraints. The only difference between the three MD regions is that, while the atoms in the Inner MD Region are subject only to their interatomic forces, the Interface and Surface MD Regions serve the added purpose of facilitating the application of external forces involved in the ESCM procedure.

The addition of a FEM domain permits a large reduction in the computational cost of simulations by replacing the atomistic representation with a continuum model in those parts of the system where the deformation gradients are small and atomic-level resolution is not necessary. The current application uses the FEM domain to simulate an extended material model such that the elastic deformation and load transfer due to applied far-field boundary conditions are accurately transferred to the Inner MD Region. The continuum field is currently assumed to be static with linear elastic material properties but other applications of ESCM might require the incorporation of nonlinear material behavior, such as plasticity or general dynamic response, where nonlinear processes generated in the Inner MD Region can be propagated into the continuum.

\section{$2.2 \mathrm{MD} / \mathrm{FE}$ interface}

The main role of the $\mathrm{MD} / \mathrm{FE}$ Interface is to provide a computational linkage between the MD region and FEM domain. The atoms that surround a given FE node at the 
interface are partitioned to form a cell in the Interface MD Region, called an interface volume cell (IVC), as shown in Figure 2. A similar partitioning is also applied to the Surface MD Region, forming surface volume cells (SVCs). The IVCs compute averaged MD displacements at their mass center that are then prescribed as displacement boundary conditions to the associated interface finite element nodes. The IVCs need not coincide in size or shape with the finite element to which the FE node belongs. In the model described in this paper, the IVCs are formed through a Voronoi-type construction [17] by selecting those atoms with a common closest finite element node.

Typically, one finite element at the interface encompasses a region of several hundred to several thousand atoms. A lower bound for the number of atoms associated with each finite element node is determined by the requirement of obtaining a minimally fluctuating average of atomic displacements and minimizing the magnitude of generated gradients in the MD region bordering the FEM domain. With an effective average at this scale, the discreteness of the atomic structure is homogenized enough so that the FEM domain responds to the atomistic region as an extension of the continuum.

During the coupled MD-FEM simulation, a spatial average within each $k^{\text {th }}$ IVC is performed to yield the center of mass displacement, $\vec{\delta}_{C M, k}^{M D}$, which is further averaged over a certain period of $M$ MD time steps to yield the statistical displacement vector, $\vec{\delta}_{I, k}^{M D}$

$$
\vec{\delta}_{I, k}=\left\langle\vec{\delta}_{C M, k}\right\rangle_{t}=\frac{1}{M} \sum_{j=1}^{M}\left(\vec{r}_{C M, k}\left(t_{j}\right)-\vec{r}_{C M, k}(0)\right)
$$

In the above expression, $\vec{r}_{C M}\left(t_{j}\right)=\frac{1}{N_{k}} \sum_{i=1}^{N_{k}} \vec{r}_{i}\left(t_{j}\right)$ is the center of mass of the $k^{\text {th }}$ IVC containing $N_{k}$ atoms at positions $\vec{r}_{i}$ at time $t_{j}$ of the $j^{\text {th }}$ MD step. The mass center displacement, $\vec{\delta}_{C M, k}$, in Equation (1) is calculated relative to the initial zero-displacement position of the $k^{\text {th }}$ IVC, $\vec{r}_{C M, k}(0)$. The determination of this initial position is discussed in Appendix A. In turn, the IVCs distribute reaction forces from the interface finite element nodes as external forces applied to the corresponding atoms within the IVC. 


\subsection{Surface MD Region}

In order for the MD domain to deform freely in response to applied reaction forces, it is modeled using free surface boundary conditions as discussed in $[13,14]$. However, the existence of a free surface introduces several undesirable effects in the MD system. First, it creates surface tension forces that must be removed to avoid distorting the MD response. Second, because atoms at or near the free surface do not have a complete set of interacting neighboring atoms, the coordination number of the surface atoms is reduced so they are less strongly bonded to the surrounding atomic field than those within the interior. Under sufficiently large reaction forces, these atoms may be separated from the surface layer causing a surface degradation within the MD domain. To mitigate these free surface effects and to stabilize the atoms in the Interface MD Region, an additional volume of outlying atoms constituting a Surface MD Region is introduced as shown in Figure 2.

While the Surface MD Region eliminates the free surface effects within the Inner MD Region, it also introduces an undesirable fictitious stiffness that elastically constrains the deformation of the Inner MD Region. The separate effects of surface tension and the fictitious stiffness cannot be computed independently. However, their combined effect may be defined as a resultant force, $\vec{f}_{s}$, which acts at the boundary between the Surface MD Region and the Interface MD Region, and is given by the sum of two components expressed as

$$
\vec{f}_{s}=\vec{\xi}+\vec{\tau}
$$

In Equation (2), $\vec{\xi}$ is the elastic reaction of the Surface MD Region under deformation, and $\vec{\tau}$ is the force that results from the surface tension. Both forces are shown in Figure 3.

A way to mitigate both the surface tension and the elastic response of the Surface MD Region is to estimate and compensate for the force $\vec{f}_{s}$. In the ideal case, when $\vec{f}_{s}$ is fully compensated, the Surface MD Region acts as if it possesses zero stiffness and 
experiences no surface tension, thereby mitigating spurious influences on the Inner MD Region. Subdividing the Surface MD Region into a number of SVCs helps to follow the variations of $\vec{f}_{s}$ along the perimeter of the Interface MD Region. For convenience, the partitioning of SVCs can be made to follow the IVC partitioning of the Interface MD Region. The resultant force is then calculated individually for each SVC. To compensate for $\vec{f}_{s}$, a counterforce, $\vec{f}_{c}$, is computed along the IVC/SVC interface and then distributed over the atoms of each SVC in a similar manner as the nodal reaction forces are applied to the IVCs of the Interface MD Region. The calculation of the counterforce, $\vec{f}_{c}$, is presented in Appendix B.

\subsection{Phonon Damping}

Both the IVCs and SVCs serve the additional role of providing a dissipative damping mechanism for phonons propagating into the interface. Potential sources of phonon generation are the application of the FEM reaction forces to the IVCs and the resonant elastic oscillations in the dynamic MD region. Phonons can also be generated from within the Inner MD Region as a result of simulated atomistic processes. In the current application of ESCM, these oscillations must be damped in order to achieve equilibrium with the static FEM domain. A number of different damping schemes have been addressed in the literature. Holian and Ravelo [18], and more recently, Schäfer et al. [19], found that applying linearly increasing viscous damping to the atoms in a region surrounding the center of the MD system can effectively absorb the intense phonon waves coming from a propagating crack. In this scheme, a friction force, $\vec{\eta}$, is given by

$$
\vec{\eta}=-\chi \vec{v}
$$

and is applied to the atoms of the damped region in proportion to the atom's velocity, $\vec{v}$, and an appropriately chosen viscous coefficient $\chi[19,20]$. The method is efficient and simple to implement. Its drawback is that it erroneously decreases the local temperature 
in the damping region resulting in undesirable strain gradients because of thermal contraction.

To avoid disturbing the thermal field, the damping used in the present method is based on a modified form of Equation (3), where, instead of being proportional to each individual atom's velocity, $\vec{\eta}$ is set proportional to the group velocity of the mass center of a certain volume of $N$ atoms. The frictional force, $\vec{\eta}$, and the group velocity of the mass center, $\vec{v}_{c m}$, are given by

$$
\vec{\eta}=-\chi \overrightarrow{\mathrm{v}}_{c m} ; \quad \overrightarrow{\mathrm{v}}_{c m}=\frac{1}{N} \sum_{i=1}^{N} \overrightarrow{\mathrm{v}}_{i} .
$$

By controlling the size of this damping region, one can damp phonons of wavelengths larger than the volume size while leaving the shorter wavelengths associated with random thermal fluctuations unaffected because their contribution to the group velocity averages to zero. Phonons introduced by the FE mesh cannot have wavelengths smaller than the distance between the interface FE nodes. Thus, it is convenient to choose both the Interface and Surface MD Regions as the volumes in which damping is applied.

To ensure a gradual stepwise increase of the viscous coefficient, $\chi$, from zero to $\chi_{\max }$, the Interface and the Surface MD Regions are additionally subdivided into $K$ layers of atoms parallel to the interface line. The layer thickness is equal to the maximum interaction distance of the applied interatomic potential $(0.65 \mathrm{~nm})$. In each layer, $\chi$ increases by a fixed amount $\Delta \chi$ starting from $\Delta \chi$ for the innermost layer, which neighbors the Inner $\mathrm{MD}$ region, to $K \Delta \chi=\chi_{\max }$ for the outermost layer, at the edge of the $\mathrm{MD}$ domain. The damping volumes used for calculating the group velocity in Equation (4) are the cross-sections of the layers with the IVCs and SVCs.

A discussion of criteria for the selection of the value of the viscous coefficient, $\chi$, can be found in [19]. In those instances where viscous wave damping is not adequate (e.g., collisional dynamics), the more precise non-reflective boundary condition techniques discussed in [20-22] may be implemented. 


\subsection{MD-FEM coupling}

The MD-FEM coupling in the ESCM is achieved through an iterative equilibration scheme between the MD region and the FEM domain. In this scheme, iterations begin with displacements at the $\mathrm{MD} / \mathrm{FE}$ Interface that are calculated as statistical averages over the atomic positions within each IVC and averaged over the time of the MD analysis. These average displacements are then imposed as displacement boundary conditions, $\left\{\vec{\delta}_{I}\right\}$, on the FEM domain. The resulting FEM BVP is then solved to recover new interface reaction forces, $\left\{\vec{R}_{I}\right\}$, resulting from the applied interface displacements and any imposed far-field loading. The new interface reaction forces, $\left\{\vec{R}_{I}\right\}$, are then distributed to the atoms in the IVCs, thus defining new constant traction boundary conditions on the MD system. Between the FEM solution updates, the traction boundary conditions are constant and applied to the MD region to ensure that the elastic field from the FEM domain is correctly duplicated in the atomistic region. The MD-FEM iteration cycle repeats until a stable equilibrium of both displacements and forces between the atomistic and continuum material fields is established at the interface.

The stiffness of the material in the FEM domain is described through a partitioning of the global stiffness matrix into a set of stiffness submatrices, $\left[K_{\alpha \beta}\right]$, with $\alpha, \beta=V, F, I$ indicating: $V$ as variables within the interior of the FEM domain; $F$ as far-field variables, and $I$ as interface variables. Using these definitions, the static continuum equations of state at the $n^{\text {th }}$ FEM update at time $t_{n}$ are given by

$$
\left[\begin{array}{lll}
{\left[K_{V V}\right]} & {\left[K_{V F}\right]} & {\left[K_{V I}\right]} \\
{\left[K_{F V}\right]} & {\left[K_{F F}\right]} & {\left[K_{F I}\right]} \\
{\left[K_{I V}\right]} & {\left[K_{I F}\right]} & {\left[K_{I I}\right]}
\end{array}\right]\left\{\begin{array}{l}
\left\{\delta_{V}\left(t_{n}\right)\right\} \\
\left\{\delta_{F}\left(t_{n}\right)\right\} \\
\left\{\delta_{I}\left(t_{n}\right)\right\}
\end{array}\right\}=\left\{\begin{array}{l}
\left\{R_{V}\left(t_{n}\right)\right\} \\
\left\{R_{F}\left(t_{n}\right)\right\} \\
\left\{R_{I}\left(t_{n}\right)\right\}
\end{array}\right\}
$$

To ensure that the FEM domain has the same elastic properties as the MD system, the stiffness terms in the $\left[K_{\alpha \beta}\right]$ submatrices are calculated from the anisotropic elastic constants derived from the MD interatomic potential [23] at the same temperature as the MD simulation. The FE model is subjected to two types of displacement boundary 
conditions: (1) the far-field displacements $\left\{\delta_{F}\right\}$, which define the load over the entire coupled MD-FEM system; and (2), the interface displacements $\left\{\delta_{I}\right\}=\left(\vec{\delta}_{I}^{1}, \vec{\delta}_{I}^{2}, \ldots, \bar{\delta}_{I}^{k}\right)$, which represent the deformation response of the MD system at the $1^{\text {st }}, 2^{\text {nd }}, \ldots$, and $k^{\text {th }}$ IVC.

The solution for the unknown displacements in the interior of the FEM domain, $\left\{\delta_{v}\right\}$, is given by

$$
\left\{\delta_{V}\left(t_{n}\right)\right\}=\left[K_{V V}\right]^{-1}\left(\left\{R_{V}\left(t_{n}\right)\right\}-\left[K_{V F}\right]\left\{\delta_{F}\left(t_{n}\right)\right\}-\left[K_{V I}\right]\left\{\delta_{I}\left(t_{n}\right)\right\}\right)
$$

which allows the calculation of the interface reaction forces, $\left\{R_{I}\left(t_{n}\right)\right\}=\left(\vec{R}_{I}^{1}, \vec{R}_{I}^{2}, . . \quad \vec{R}_{I}^{k}, ..\right)$ of the $1^{\text {st }}, 2^{\text {nd }}, \ldots$, and $k^{\text {th }}$ IVC to be obtained from

$$
\left\{R_{I}\left(t_{n}\right)\right\}=\left[K_{I V}\right]\left\{\delta_{V}\left(t_{n}\right)\right\}+\left[K_{I F}\right]\left\{\delta_{F}\left(t_{n}\right)\right\}+\left[K_{I I}\right]\left\{\delta_{I}\left(t_{n}\right)\right\}
$$

together with the far-field forces of constraint

$$
\left\{R_{F}\left(t_{n}\right)\right\}=\left[K_{F V}\right]\left\{\delta_{V}\left(t_{n}\right)\right\}+\left[K_{F F}\right]\left\{\delta_{F}\left(t_{n}\right)\right\}+\left[K_{F I}\right]\left\{\delta_{I}\left(t_{n}\right)\right\}
$$

The dynamics of an atom $i$ of mass $m^{(i)}$ at position $r^{(i)}$ in the embedded MD regions is described by Newton's equations of motion

$$
\begin{array}{ll}
m_{i} \ddot{\bar{r}}_{i}=\vec{f}_{i} ; & i \in \text { Inner MD Region } \\
m_{i} \ddot{\ddot{r}}_{i}=\vec{f}_{i}+\vec{R}_{I}^{k} / N_{I}^{k}-\chi \overrightarrow{\boldsymbol{v}}_{c m}^{k} ; & i \in(I V C)_{k} \text { Interface MD Region } \\
m_{i} \ddot{\bar{r}}_{i}=\vec{f}_{i}+\vec{f}_{c}^{k} / N_{S}^{k}-\chi \overline{\mathbf{v}}_{c m}^{k} ; & i \in(S V C)_{k} \text { Surface MD Region }
\end{array}
$$

The atoms in the Inner MD Region experience only the atomic force $\vec{f}_{i}=\sum_{j} \vec{f}^{(i, j)}$ resulting from their $j^{\text {th }}$ neighbors. The term $\vec{f}^{(i, j)}$ is expressed by Equation (B4) as shown in Appendix B. The atoms in the interface region, assigned to a given $k^{\text {th }}$ IVC, are subjected to the additional external force, $\vec{R}_{I}^{k}$ (Equation (7a)), which is distributed over 
the number of contained atoms, $N_{I}^{k}$. The atoms in the Surface MD Region belonging to a given $k^{\text {th }}$ SVC experience the additional counterforce, $\vec{f}_{c}^{k}$, which is distributed over the $N_{S}^{k}$ atoms contained in their volume. In order to maintain the continuity of forces between adjacent cells, the force distribution is interpolated with a linear (or higher order) interpolation between atomic positions as a function of each atom's distance from the mass center of its associated cell. In the present study, a simple linear interpolation was applied. For the equations governing the Interface and the Surface MD Regions in Equation (8), the viscous friction force, $\chi \overrightarrow{\mathbf{v}}_{c m}^{k}$, defined in Equation (4), is applied uniformly to the atoms contained within the IVCs and SVCs.

During the MD integration of Equation (8) for a period $\Delta t_{\mathrm{M}}=M \Delta t$, where $M$ is the number of time steps and $\Delta t$ is the duration of the time step, the new average displacements $\left\{\delta_{I}\left(t_{n+1}\right)\right\}$ are computed from Equation (1). The new atomistic displacements for the next FEM update at time $t_{n+1}=t_{n}+\Delta t_{M}$ are reapplied in Equations (6) and (7a) to calculate the next iterative update of the recovered forces, $\left\{R_{I}\left(t_{n+1}\right)\right\}$. During the same time interval, the compensation forces $\left\{f_{c}(t)\right\}$ are also evolving through Equation (B15) (in Appendix B). The period $\Delta t_{\mathrm{M}}$ is selected by a determination of the convergence rate to a state of dynamic equilibrium between the MD region and the FE domain. Applying a suitable damping force (Equation 4) at the MD side of the interface ensures a faster convergence rate. The algorithm for the entire coupled simulation is summarized in Figure 4.

Indication for convergence between the MD and FEM domains is the convergence of the interface forces $\left\{R_{I}\right\}$ and displacements $\left\{\delta_{I}\right\}$ to equilibrium steady-state values. This convergence can be achieved only if the MD system can reach a dynamic force balance with the surrounding FEM system. A convergence criteria will be derived based on the force balance between the atomic forces in the MD system and the reaction forces in the FEM domain. At equilibrium, the averaged in time motion of the IVC mass centers is zero, or

$$
\left\langle\dot{\vec{r}}_{C M}^{k}\right\rangle_{t}=\left\langle\overline{\boldsymbol{v}}_{C M}^{k}\right\rangle_{t}=0,
$$


and the change of the averaged total momentum, $\left\langle\Delta p_{k}\right\rangle_{t}$, of any $k^{\text {th }}$ IVC due to the FEM reaction force for the period of the MD simulation of $M$ time steps is also zero, or

$$
\Delta \overrightarrow{\mathrm{p}}_{\mathrm{k}}=\sum_{\mathrm{n}=1}^{\mathrm{M}} \sum_{\mathrm{i}=1}^{\mathrm{N}_{\mathrm{I}}^{\mathrm{k}}} \mathrm{m}_{\mathrm{i}} \ddot{\overline{\mathrm{r}}}_{\mathrm{i}}\left(\mathrm{t}_{\mathrm{n}}\right) \Delta \mathrm{t}=0 .
$$

Performing the same double summation on the second equation in Equation (8) results in

$$
\frac{1}{M} \sum_{m=1}^{M} \sum_{i=1}^{N_{I}^{k}} \vec{f}_{i}\left(t_{m}\right)=\left\langle\sum_{i=1}^{N_{I}^{k}} \vec{f}_{i}\right\rangle_{t}=-\vec{R}_{I}^{k}
$$

which states that, at equilibrium and in accordance with Newton's $3^{\text {rd }}$ law, the FEM reaction force becomes equal and opposite to the average MD atomic force in the corresponding $k^{\text {th }}$ IVC. Equation (10) thus expresses the establishment of static force equilibrium between the MD system and the FEM domain and can be used as a convergence criteria for the iterative MD-FEM coupling procedure.

A discussion of practical issues regarding model generation for applying the ESCM procedure is presented in Appendix A.

\section{NUMERICAL VERFICATION OF THE ESCM}

\subsection{The simulation models}

Four test cases are considered to investigate the behavior of ESCM. First, the effectiveness of the application of compensation forces for the mitigation of surface tension effects is examined. Second, the dynamic behavior of the MD system is explored by varying the rate and sequence of applied external loads. Third, the stress-strain continuity between the MD region and the FEM domain is assessed through comparison with an exact solution of an elastically deformed plate with a circular hole. Fourth, a simulation of the propagation of an edge crack through the FEM domain into the MD system is performed to determine the suitability of ESCM for solving problems related to crack growth. 
The model geometry used in all of the verification studies is shown in Figure 5. This model consists of a circular Inner MD Region of diameter, $d_{M D}$, that is embedded in a larger exterior square FEM domain of elastic material with a side dimension of $d_{F E}=$ $20 d_{M D}$. A general discussion of issues related to model construction in applying ESCM is presented in Appendix A, while the specifics of the MD and FE models will be discussed next.

\subsubsection{The MD model}

The material for the simulation models was chosen to be a perfect crystal of aluminum. The atomic properties of aluminum were represented by the embedded atom model (EAM) potential of Mishin et al. [24], which was fitted to give the correct zerotemperature lattice constant, $a_{\mathrm{o}}=0.405 \mathrm{~nm}$, elastic constants, cohesive energy, vacancy formation energy, etc. For accurate coupling, material properties of the FE model are obtained directly from the EAM interatomic potential.

The first three test simulations, presented in Sections 3.2.1 to 3.2.3, use a common MD system which will be described here. The forth test is performed on a bicrystal MD model which will be described in Section 3.2.4. For the first three tests, the MD domain is constructed as a circular disk of monocrystalline aluminum with its main crystallographic axes [lll 00$],\left[\begin{array}{lll}0 & 1 & 0\end{array}\right]$, and [ $\left[\begin{array}{lll}0 & 0 & 1\end{array}\right]$ oriented along the $x-, y$ - and $z-$ directions, respectively. The MD system is simulated with periodic boundary conditions along the $z$-direction and with free surface boundary conditions along its perimeter. These boundary conditions allow the MD domain to deform in an unconstrained manner in the $x-y$ plane under the external reaction forces from the FEM domain while maintaining constant zero pressure along the z-direction using the Parrinello-Rahman constantpressure simulation technique [25]. Constant temperature is maintained by applying the Nose-Hoover thermostat [26] in the Inner MD Region only. The thickness of the plate along the $z$-axis is equal to $h=5 a_{\mathrm{o}} \approx 2.0 \mathrm{~nm}$ (Figure 5). Though very thin, the MD system mechanically behaves as an infinitely thick plate due to the applied periodic boundary conditions along the $z$ - direction. The test simulations were performed at near zero temperature $(T=10 \mathrm{~K})$ to minimize the thermal noise, and at room temperature $(T=300$ 
$\mathrm{K})$ to demonstrate ESCM for more practically relevant situations in which thermal effects are important. For the models used in the present work, effective viscous wave damping in the Interface and the Surface MD Regions was achieved by setting $\chi_{\max }=3 \mathrm{eVps} / \mathrm{nm}^{2}$. For this $\chi$ and a damping layer with a width of $0.65 \mathrm{~nm}$, the effective average temperature in the damping volumes decreased by only $10 \%$ compared to the bulk temperature.

Four different models were prepared with the diameter of the circular MD system varying from $22 \mathrm{~nm}$ to $164 \mathrm{~nm}$. Reference positions of the IVC mass centers, $\vec{r}_{C M}(0)$, were determined using methods outlined in Appendix A. The width of the Surface MD Region, defined at the free surface of the MD system, was fixed at $2 \mathrm{~nm}$.

\subsubsection{The FE model}

The elastic continuum region was modeled using 8-node hybrid-stress hexahedral finite elements that have a reduced sensitivity to mesh distortion compared to standard displacement-based elements, and allow explicit stiffness coefficients to be analytically derived, thereby minimizing their computational requirements [27,28]. The elastic constants in the material constitutive matrix were derived from the interatomic potential for pure aluminum at $\mathrm{T}=10 \mathrm{~K}$. The values were averaged for uniaxial stresses from 100 to $500 \mathrm{MPa}$, accounting for the non-linear material properties, as: $\mathrm{C}_{11}=112.7 \mathrm{GPa}, \mathrm{C}_{12}=$ $59.4 \mathrm{Gpa}$, and $\mathrm{C}_{44}=30.6 \mathrm{GPa}$. These values differ by only $3 \%$ from the static, zero Kelvin elastic constants reported for this potential in [24].

The continuum finite element model contains an open inner region of diameter $d_{\mathrm{MD}}$, within which the atomistic domain is embedded. Along its perimeter, 80 nodes at $z=$ $+h / 2$ and 80 nodes at $z=-h / 2$ were placed to form $160 \mathrm{FE}$ interface nodes to communicate with the embedded MD system.

The dimensions of the FE mesh, $d_{F E}, d_{M D}$ and $h$ as shown in Figure 5 were initially defined through the proportions of $d_{F E}: d_{M D}: h=20: 1: 1$. Then, a direct scaling of the FE nodal coordinates was performed such that the dimensions $d_{\mathrm{MD}}$ and $h$ matched the 
dimensions of the MD system. Finally, a second scaling of the FEM system was performed to preserve the outer dimension ratio $d_{F E}: d_{M D}=20: 1$.

\subsection{Numerical test results and analyses}

The four test cases selected to interrogate the essential features of the ESCM are presented in the following sections. Discussions assessing results and details of the analyses are included to thoroughly investigate the verification simulations.

\subsubsection{Verification of the surface tension and the Surface MD Region stiffness compensation}

The purpose of this simulation is to estimate the magnitude of the surface forces, their effect on coupling the two computational domains, and the ability of the compensation procedure to mitigate both surface tension effects and the spurious constraint of the Surface MD Region stiffness. The simulation is performed for the case of a homogeneous perfect crystal of aluminum. Because the effect of the surface tension is expected to be relatively weak, the temperature of the simulations was kept at $T=10 \mathrm{~K}$ to minimize the thermal noise and to increase the sensitivity of the force and pressure calculations.

The pressure inside the system due to surface tension is defined as the radial component of the stress tensor, $p_{\mathrm{s}}=\sigma_{r r}$, averaged over an isolated MD system with free surface boundary conditions in the $x$ - and $y$-directions, and periodic, zero pressure boundary conditions applied in the z-direction. The virial definition of stress [29] inside the MD system was used. The surface pressure increases from 10 to $80 \mathrm{MPa}$ with $d_{M D}$ decreasing from 164 to $22 \mathrm{~nm}$ as presented in Figure 6. The expected dependence of $p_{s}$ on surface tension, $\gamma,\left(p_{s}=2 \gamma / d_{M D}\right)$ for a cylindrical nanoparticle [30] is well reproduced. The surface tension, estimated from the slope of a linear fit to the results in Figure 6 as $\gamma$ $=0.9 \mathrm{~J} / \mathrm{m}^{2}$, is found to be in the range of the calculated surface energies for the interatomic potential used $\left(\gamma_{\mathrm{s}}=0.87,0.943\right.$, and $1.006 \mathrm{~J} / \mathrm{m}^{2}$ for (111), (100) and (110) surfaces, respectively [24]). While the values for $p_{\mathrm{s}}(<0.1 \mathrm{GPa})$ are relatively small for a 
MD simulation (where typical loads are of the order of $1 \mathrm{GPa}$ ), particularly for small $\mathrm{MD}$ systems, its contribution should not be neglected.

The effect of applying a counterforce to compensate for the surface tension is shown in Figure 7 for the case of $d_{\mathrm{MD}}=44 \mathrm{~nm}$. When no surface compensation force is applied, the internal pressure, $p_{\mathrm{s}}$, gradually increases from zero due to the evolving effect of surface tension forces and approaches the value of $p_{\mathrm{s}}=41.5 \mathrm{MPa}$. In contrast, repeating the same simulation with the compensation force applied quickly reduces $p_{\mathrm{s}}$ to near zero. The short initial increase in the value of $p_{\mathrm{s}}$ observed during the first $10 \mathrm{ps}$ is a result of the iteration procedure (Equation (B15)) for adjusting the counterforce, $f_{\mathrm{c}}$, having not yet reached convergence and the compensation being not yet complete. The compensation becomes complete approximately 20 ps after the beginning of the simulation.

Under deformation, the stiffness of the Surface MD Region creates an elastic reaction force that adds to the surface tension. As explained in Section 2.3, the iteration procedure for $f_{\mathrm{c}}$ was developed to compensate for both effects. Figure 8 shows the combined effect of surface tension and the elastic stiffness of the Surface MD Region on the equilibrium stress state of the Inner MD Region for four different MD system sizes ranging from $d_{\mathrm{MD}}$ $=22$ to $164 \mathrm{~nm}$ at $0.5 \%$ far-field strain. As the dynamic response of the MD system strongly depends on its size, to make the simulations comparable, the time $t$ is rescaled by an estimated relaxation time, $t_{0}$, for each system size. The relaxation time is defined in the standard way as the period required for an exponential variable to decrease by $1 / \mathrm{e}(0.386)$ of its initial value. In Figure 8, the exponential variable is the deviation of the current stress, $\sigma_{x x}(t)$, at time, $t$, from its equilibrium value, $\sigma_{\infty}$, established at $t / t_{\mathrm{o}}=5$ with no counterforce, $\vec{f}_{c}(t)$

$$
\sigma_{\infty}-\sigma_{x x}(t)=e^{-t / t_{o}}
$$

The relaxation time, $t_{0}$, increases with increasing size and mass of the MD domain. For $d_{M D}=22,44,84$ and $164 \mathrm{~nm}$ (containing 47 600, 190 300, 693 400, and 2641000 atoms, respectively), a curve fit to Equation (11) gave $t_{\mathrm{o}}=5.1,19.5,61$, and $200 \mathrm{ps}$, respectively.

Initially $\left(t / t_{0}<5\right)$, no counterforce was applied in the Surface MD Region and $\sigma_{x x}$ equilibrated to a lower level compared to the far-field stress, $\sigma_{o}$, of a homogeneously 
strained plate. The stress due to the Poisson contraction of the unconstrained boundaries $\left(y= \pm d_{F E} / 2\right), \sigma_{y y}$, also deviated from the expected zero level. The reason for these deviations is the combined effect of surface tension and the elastic stiffness of the Surface MD Region. The deviations of both $\sigma_{x x}$ and $\sigma_{y y}$ decrease proportionally to the increase of $d_{\mathrm{MD}}$, as expected because of the decreasing surface-to-volume ratio. When the counterforce is applied $\left(t / t_{\mathrm{o}}>5\right)$, the effect of the spurious forces in the Surface MD Region for all of the tested MD systems of $d_{\mathrm{MD}}$ from 22 to $164 \mathrm{~nm}$ is almost entirely negated.

\subsubsection{Simulation of the dynamics of the coupled MD-FEM system}

The dynamic behavior of the coupled MD-FEM system in the simplest case of a uniformly loaded homogeneous aluminum plate is depicted in Figure 9. The figure presents the stress response of the Inner MD Region to the applied far-field strain. The system is the same as that used for the results in Figure 7, with $d_{M D}=44 \mathrm{~nm}$. In this numerical test, the prescribed strain of $\varepsilon_{x x}=0.5 \%$ was reached in two ways: first, by an instantaneously applied far-field strain of $0.5 \%$ at the outer-boundaries of the FEM domain, and second, by five consecutive increments of $0.1 \%$ each. In both cases, the length of the MD iteration simulation was $\Delta t_{\mathrm{M}}=M \Delta t=1 \mathrm{ps}$ with $M=500$ and $\Delta t=2 \mathrm{fs}$.

The tensile component of the stress in the MD system, $\sigma_{x x}$, converges to nearly the same value for both cases shown in Figure 9 and is very close to the far-field stress of the FEM domain, $\sigma_{o}\left(\sigma_{x x} \rightarrow 354 \mathrm{MPa}\right.$ and $\left.\sigma_{o}=358.5 \mathrm{MPa}\right)$. Similarly, $\sigma_{y y}$ quickly relaxes to zero after a temporary jump in response corresponding to each increase in the applied farfield strain. The test shows that the state of equilibrium, wherein the MD system is in force balance with the FEM domain, is obtained with little dependence on step size for monotonic loading up to $0.5 \%$ strain.

After each strain increment of $0.1 \%$, the MD system reached equilibrium with the FEM domain within approximately $25 \mathrm{ps}$, which is consistent with the estimate for $t_{\mathrm{o}}=$ $19.5 \mathrm{ps}$ in Figure 7 for $d_{\mathrm{MD}}=44 \mathrm{~nm}$. This time was approximately the same as for the instantaneous jump to $0.5 \%$. The relatively large response time observed in Figures 8 and 9 indicates that using a static FE model (Equation (5)) for the continuum part of the 
system is not suitable for simulating processes in the MD system that are evolving faster than $t_{\mathrm{o}}$.

3.2.3 Test for stress-strain continuity over the MD and the FEM regions: elastically deformed plate with a circular hole

To assess the capability of the ESCM for generating compatible stress-strain fields in the elastic continuum domain and the MD atomistic region, the classic example of a plate with a circular hole subjected to uniaxial loading was used. In addition to having an exact elasticity solution for the slightly anisotropic material properties used [31,32], this model is particularly convenient for two reasons. First, it provides large stress variations (from zero to $2.82 \sigma_{o}$ ) around the hole, which can be used to test the continuity of the stress field at the $\mathrm{MD} / \mathrm{FE}$ interface in the case of large stress gradients. Secondly, keeping the peak stress, $2.82 \sigma_{o}$, well below the theoretical elastic limit of the material (recently estimated for the applied interatomic potential to be between 3 and $5 \mathrm{GPa}$ [33]) prevents the occurrence of any plastic mechanisms in the MD region, which is not addressed in the present study, and a static elastic equilibrium can be achieved everywhere in the system.

For comparison, an equivalent fully continuum three-dimensional anisotropic FE model was also simulated with a hole of radius $20 \mathrm{~nm}$. This model was generated within a square FE mesh of dimension $1.6 \mu \mathrm{m}$ by $1.6 \mu \mathrm{m}$ and having the same elastic properties (derived from the interatomic potential) as the FE part of the coupled MD-FE model.

In both models, the square plate was deformed at $0.5 \%$ uniaxial strain along the $x$ direction through displacement-controlled boundary conditions imposed on the outer sides of the FEM system $800 \mathrm{~nm}$ away from the hole in the MD domain. At this strain, the far-field stress, estimated in Figure 9, is $\sigma_{0}=358.5 \mathrm{MPa}$.

Starting from an undeformed MD region, the coupled MD-FEM iterative simulation was performed until equilibrium was established between the MD domain and the FEM domain whereby $\left\{\delta_{I}\right\}$ and $\left\{R_{I}\right\}$ converged to constant values. The stress field for $\sigma_{x x}, \sigma_{y y}$, and $\sigma_{x y}$ stress components of the coupled MD-FEM system was calculated and compared with the fully continuum FEM solution that was obtained using the ABAQUS software package [34]. This comparison is shown in Figure 10. 
The stress profiles for $\sigma_{x x}$ and $\sigma_{y y}$ taken along sections coincident with the $x$ - and $y$ axes passing through the center of the hole as shown in Figure 10(a) are presented in Figures 11(a) and 11(b), respectively. The continuity of the stress profiles is well preserved at the $\mathrm{MD} / \mathrm{FE}$ interface apart from fluctuations that result from chaotic atomic thermal vibrations. Additionally, the stress profiles of the coupled model closely follow the stress profiles of the fully continuum FEM analysis of the equivalent model. The largest discrepancy is seen for the tangential component of the stress ( $\sigma_{y y}$ along the $x$ profile in Figure 11(a) and $\sigma_{x x}$ along the $y$-profile in Figure 11(b)) in the MD system, which deviates substantially from the continuum prediction when approaching the surface of the hole. As shown in Figure 11(b), the continuum solution closely approaches the theoretical value of $2.82 \sigma_{o}$ that is calculated for the slightly anisotropic material used. One reason for this discrepancy may be the definition of virial stress, which gives poor convergence and erroneous estimates near free surfaces [35]. But more likely it is that the continuum model does not correctly account for the nature of the surface tension, which results from the occurrence of missing bonds between the atoms at the free surface. From the previous analysis (Figure 3), it was found that the normal pressure of a free surface with a curvature radius of $40 \mathrm{~nm}$ is $p_{\mathrm{s}}=45 \mathrm{MPa}$. This value agrees well with the normal component of the stress estimated from the MD simulation ( $\sigma_{x x}$ along the $x$-profile in Figure 11(a), and $\sigma_{y y}$ along the $y$-profile in Figure 11(b)) at the edge of the hole, where the corresponding FEM solution approaches zero.

\subsection{Example of an edge crack simulation along a grain boundary in aluminum}

An important application of the ESCM technique described in this paper is the simulation of atomistic processes related to damage. A typical example is the tip of an

edge crack, where the idealized elastic stress field decreases as $1 / \sqrt{r}$ and extends to a distance $r$ from the tip. The crack tip stress field is much larger than a MD system can computationally simulate. A coupled MD-FE model for this example is presented in Figure 12, where the MD system is used to atomistically simulate the plastic zone at the crack tip, and the FEM domain is used to provide the continuum elastic boundary 
conditions of a far-field tensile strain of $\varepsilon_{y y}=2 \%$ applied along the $y$-direction. To investigate the applicability of ESCM at higher temperatures, the simulation was carried out at room temperature $(T=300 \mathrm{~K})$.

The dimensions of the system are: $h=2.9 \mathrm{~nm}, d_{\mathrm{MD}}=45 \mathrm{~nm}$, and $d_{\mathrm{FE}}=900 \mathrm{~nm}$. The MD system represents a bi-crystal with a high-angle grain boundary formed between the two crystals along which the edge crack propagates. In the selected coordinate system of

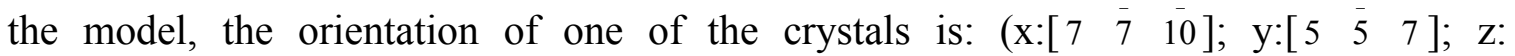

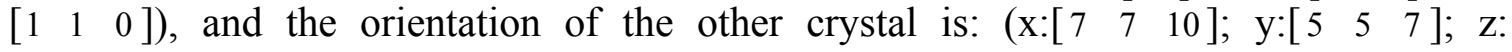
$\left.\left[\begin{array}{lll}1 & 1 & 0\end{array}\right]\right)$. In this way, both crystals are mirror images of each other relative to the crystallographic plane $\left\{\begin{array}{lll}5 & 5 & 7\end{array}\right\}$, which becomes the plane of the grain boundary (GB) between them. The GB thus formed is a $<1 \quad 1 \quad 0>\sum 99$ symmetric tilt GB. Crack propagation along this GB has been extensively studied by MD simulations [36-38] at a cryogenic temperature of $T=100 \mathrm{~K}$.

In the stresses discussed in [36-38], it was found that, while in one direction the crack becomes blunted by deformation twinning, in the opposite direction it propagates in a brittle-like manner with very little dislocation emission. The latter direction has been chosen as the propagation direction for the edge crack of the simulation in Figure 12. The simulation using the ESCM approach performed at room temperature showed higher dislocation plasticity than at $T=100 \mathrm{~K}$ [36-38]. The remaining problem is how to transfer this plasticity to the FEM continuum. Some work related to this issue has been started independently by Curtin et al. [3], Shilkrot et al. [8], and Qu et al. [9], where the CADD coupling methodology has been developed to follow and transmit dislocations between the atomistic and continuum regions. However, that methodology is not employed here.

What is essential in the example shown in Figure 12 is that the ESCM approach preserves the continuity of the stress - strain field at the MD-FEM interface even for a dynamic problem such as crack propagation simulated at room temperature. The depicted profile shows the continuity of stresses across the boundary when the crack speed is slow compared to the elastic response time of the system. In the example shown in Figure 12, 
the crack propagation speed was approximately $100 \mathrm{~m} / \mathrm{s}$ or on the order of $1 / 30$ the speed of sound.

\section{SUMMARY}

A new statistical approach to couple MD with FEM simulations, denoted the embedded statistical coupling method (ESCM), has been developed. The approach is based on solving the boundary value problem through an iterative procedure for both MD and FEM systems at their common interface. The two systems are simulated independently, and they communicate only through their boundary conditions. The FEM system is loaded by far-field loads applied to the external boundaries and along the $\mathrm{MD} / \mathrm{FE}$ interface by nodal displacement boundary conditions that are obtained as statistical averages of the atomic positions in the MD system at the mass centers of representative interface volume cells (IVCs) associated with each FE node along the interface. The MD system, in turn, is simulated under periodically updated constant traction boundary conditions that are obtained from the FEM system as reaction forces to the MD displacements at the interface. This iterative approach allows the continuity at the $\mathrm{MD} / \mathrm{FE}$ interface to be achieved at different length and time scales inherent to both systems without the need of redefining continuum quantities at the discrete atomic scale and atomic quantities at the continuum scale.

Compared to the widely used direct coupling methods, ESCM does not discretize the $\mathrm{MD} / \mathrm{FE}$ interface to atomistic scales but uses a statistical mapping of atomistic behavior to a continuum FEM representation. ESCM presents a simple and flexible technique in providing elastic boundary conditions for a MD model and eliminates some of the finitesize artifacts inherent to a purely atomistic simulation. Because of the statistical connection between the MD and the FEM systems, there is no limitation on the temperature of the atomistic system as long as the thermal corrections to the elastic properties of the continuum system are considered. 
Using static FEM calculations for the continuum part of the system, the ESCM shows excellent convergence for systems simulated under static elastic equilibrium and preserves stress continuity at the $\mathrm{MD} / \mathrm{FE}$ interface for systems exhibiting relatively slow dynamics governed by the MD simulation of the atomistic part of the system. Additional study needs to be performed to determine if the implementation of a dynamic FEM simulation can improve the dynamics of the system away from equilibrium.

In general, the method is relatively easy to implement. Any conventional FEM code, including commercial packages, can be used to solve the FEM part of the model. Only small modifications to an otherwise conventional MD code are necessary to apply the constant tractions to the MD system.

The verification simulations performed in this study demonstrated the effectiveness of the ESCM to couple atomistic and continuum modeling into a unified multiscale simulation. Several idealized test cases were analyzed to interrogate the behavior of the ESCM. First, the effectiveness of using the Surface MD Region to provide means to emulate infinite boundary conditions for the MD system was verified. Second, the dynamic behavior of the coupled MD-FEM system was explored demonstrating convergence to the same equilibrium state while varying the rate and sequence of applied loads. Third, the stress-strain continuity between the MD region and the FEM domain was validated using the model of an elastically deformed plate with a circular hole. Finally, simulating the slow propagation of an edge crack was performed to demonstrate the overall representational capability of the coupled MD-FE model in a system evolving quasi-statically. 


\section{REFERENCES}

1. Abraham FF, Broughton JQ, Bernstein N, Kaxiras E, Spanning the continuum to quantum length scales in a dynamics simulation of brittle fracture. Europhysics Letters 1998; 44:783.

2. Broughton JQ, Abraham FF, Bernstein N, Kaxiras E, Concurrent coupling of length scales: methodology and application. Physical Review B 1999; 60:2391.

3. Curtin WA, Miller RE, Atomistic/continuum coupling in computational materials science. Modeling and Simulation in Materials Science and Engineering 2003; 11:R33.

4. Park HS, Liu WK, An introduction and tutorial on multiple-scale analysis in solids. Computational Methods in Applied Mechanics and Engineering 2004; 193:1733.

5. Li X, E W, Multiscale modeling of the dynamics of solids at finite temperature. Journal of the Mechanics and Physics of Solids 2005; 53:1650.

6. Miller RR, Tadmor EB, The quasicontinuum method: overview, applications and current directions. Journal of Computer Aided Materials Design 2002; 9:203.

7. Xiao SP, Belytschko T, A bridging domain method for coupling continua with molecular dynamics. Computational Methods in Applied Mechanics and Engineering 2004; 193:1645.

8. Shilkrot LE, Miller RE, Curtin WA, Coupled atomistic and discrete dislocation plasticity. Physical Review Letters 2002; 89:025501.

9. Qu, S., Shastry, V., Curtin, W.A., and Miller, R.E., "A Finite-Temperature Dynamic Coupled Atomistic/discrete Dislocation Method," Modeling and Simulation in Materials Science and Engineering 2005; 13:1101.

10. Gumbsch P, Beltz GE, On the continuum versus atomistic description of dislocation nucleation and cleavage in nickel. Modeling and Simulation in Materials Science and Engineering 1995; 3:597.

11. Rudd RE, Broughton JQ, Coarse-grained molecular dynamics and the atomic limit of finite elements. Physical Review B 1998; 58:R5893.

12. Rudd RE, Broughton JQ, Coarse-grained molecular dynamics: nonlinear finite elements and finite temperature. Physical Review B 2005; 72:144104.

13. Steinmann, P., Elizondo, A., Sunyk, R., "Studies of validity of the Cauchy-Born rule by direct comparison of continuum and atomistic modelling," Modelling and Simulation in Materials Science and Engineering, 2007; 15:S271.

14. deCelis B, Argon AS, Yip S, Molecular dynamics simulation of crack tip processes in alpha-iron and copper, Journal of Applied Physiscs 1983; 54:4864.

15. Cheung KS, Yip S, A molecular-dynamics simulation of crack-tip extension: the brittle-to-ductile transition, Modelling Simul. Mater. Sci. Eng. 1994; 2:865-892.

16. Cleri F, Representation of mechanical loads in molecular dynamics simulations. Physical Review B 2001; 65:014107.

17. O’Rourke J, Computational Geometry in C, 2nd Edition, Cambridge University Press, 2001.

18. Holian BL, Ravelo R, Fracture simulations using large-scale molecular dynamics. Physical Review B 1995; 51:11275. 
19. Schäfer C, Urbassek HM, Zhigilei LV, Garrison BJ, Pressure-transmitting boundary conditions for molecular-dynamics simulations. Computational Materials Science 2002; 24:421.

20. Moseler M, Nordiek J, Haberland H, Reduction of the reflected pressure wave in the molecular-dynamics simulation of energetic particle-solid collisions. Physical Review B 1997; 56:15439.

21. Cai W, Koning M, Bulatov VV, Yip S, Minimizing boundary reflections in coupleddomain simulations. Physical Review Letters 2000; 85:3213.

22. Park HS, Karpov EG, Liu WK, Non-reflecting boundary conditions for atomistic, continuum and coupled atomistic/continuum simulations. International Journal for Numerical Methods in Engineering 2005; 64:237.

23. Cook RD, Malkus DS, Plesha ME, Concepts and Applications of Finite Element Analysis, Third Edition, John Wiley \& Sons.

24. Mishin Y, Farkas D, Interatomic potentials for monoatomic metals from experimental data and ab initio calculations. Physical Review B 1999; 59:3393.

25. Parrinello M, Rahman A, Polymorphic transitions in single crystals; a new molecular dynamics method. Journal of Applied Physics 1981; 52:7182.

26. Nose S, A unified formulation of the constant temperature molecular dynamics method. Journal of Chemical Physics 1984; 81:511.

27. Pian THH, Tong P, Relations between incompatible displacement model and hybrid stress model. International Journal for Numerical Methods in Engineering 1986; 22:173.

28. Saether E, Explicit determination of element stiffness matrices in the hybrid stress method. International Journal for Numerical Methods in Engineering 1995; 38:2547.

29. Cormier J, Rickman JM, Delph TJ, Stress calculation in atomistic simulations of perfect and imperfect solids. Journal of Applied Physics 2001; 89:99.

30. Huang Z, Thomson P, Shenglin D, Lattice contractions of a nanoparticle due to the surface tension: A model of elasticity. Journal of Physics and Chemistry of Solids 2007; 68:530.

31. Hirth, J.P. and Lothe, J., Theory of Dislocations, $2^{\text {nd }}$ Edition, John Wiley \& Sons, 1982.

32. Lekhnitskii, SG, Anisotropic Plates, Gordon and Breach Science Publishers, 1987.

33. Tsuru T, Shibutani Y, Atomisitc simulations of elastic deformation and dislocation nucleation in $\mathrm{Al}$ under indentation-induced stress distribution. Modeling and Simulation in Materials Science and Engineering 2006; 14:S55.

34. ABAQUS/Standard User's Manual, Hibbitt, Karlsson, and Sorensen, Inc., 2004.

35. Zimmerman JA, Jones RE, Klein PA, Bammann DJ, Webb EB, Hoyt JJ, Continuum definitions for stress in atomistic simulation. SAND Report, 2002 Sandia National Laboratory, SAND2002-8608.

36. Yamakov V, Saether E, Phillips DR, Glaessgen EH, Dynamic instability in intergranular fracture. Physical Review Letters 2005; 95:015502.

37. Yamakov V, Saether E, Phillips DR, Glaessgen EH, Molecular-dynamics simulationbased cohesive zone representation of intergranular fracture processes in aluminum. Journal of the Mechanics and Physics of Solids 2006; 54:1899. 
38. Yamakov V, Saether E, Phillips DR, Glaessgen EH, Dynamics of nanoscale grainboundary decohesion in aluminum by molecular-dynamics simulation. Journal of Materials Science 2007; 42:1466.

39. Daw MS, Baskes MI, Embedded-atom method: Derivation and application to impurities, surfaces, and other defects in metals. Physical Review B 1984; 29:6443.

\section{APPENDIX A}

\section{ESCM Model Construction}

In the ESCM approach, the statistical basis for numerically coupling the different computational frameworks provides a much less restrictive set of interfacing requirements compared to DC methods and, thus, allows a greater independence in the construction of the associated MD and FE models. This aspect of ESCM, however, results in additional preparatory work in constructing the coupled model, primarily involving the preparation of the initial state of the MD region. A schematic of a MD model is depicted in Figure A1.

The construction procedure starts with the definition of the shape and size of the MD region and FEM domain. The dimensions of the MD system are defined by the dimensions of the combined Inner, Interface, and Surface MD Regions. The dimensions of the FEM domain are selected such that the outer boundary defines the desired overall material domain and the inner boundary coincides with the IVC mass centers along the $\mathrm{MD} / \mathrm{FE}$ interface. The FE mesh conveniently provides a regular distribution of node locations to be used in a Voronoi construction of the IVCs along the MD/FE interface. Appropriate interpolation methods (e.g. linear interpolation employing finite element shape functions) must be used to accurately map quantities (e.g. interface displacements) between the IVC mass centers and the corresponding FE nodes.

It is additionally important in the construction of the ESCM model that the reference states of the MD and FEM systems coincide as closely as possible. For a static FEM domain, the displacements are zero when the applied forces are zero. For a MD region, displacements are statistical quantities that can include some statistical error in their estimate that needs to be minimized. 
Preparatory simulations of the MD system involve thermalization, equilibration, and the determination of external compensating forces. The calculation of these compensating forces is discussed in Appendix B and are required to maintain the initial atomic reference states that are necessary for this domain to exhibit the response of an infinite system influenced only by external forces when coupled to the FEM computational domain. To perform the preparatory simulations, an initial MD model of rectangular shape is chosen that is large enough such that the geometry of the desired MD region (which in the present work is a circular disc) is contained as a subset. This subset can subsequently be extracted by removing the atoms outside the desired MD region boundaries (see Figure A1).

To accurately define the zero-displacement reference state of the MD region, the system is thermally equilibrated at zero stress and simulated as a constant number of atoms, N, constant pressure, $\mathrm{P}$, and constant temperature, T, (NPT ensemble) under periodic boundary conditions ( $\mathrm{PBC}$ ) in all three dimensions. $\mathrm{PBCs}$ are necessary during this step to avoid the presence of a free surface because the surface tension would produce a pressure, $p_{\mathrm{s}}$, on the surface, resulting in erroneous zero-displacement positions for the IVC mass centers.

After achieving equilibrium, an additional MD simulation under PBCs at zero pressure and constant temperature is carried out to obtain the statistical time average of the mass centers. Larger IVCs and longer initialization times result in smaller statistical errors in the reference state because the statistical error of the estimated averages depends on the number of atoms, $N$, per IVC and the time, $t$, of the simulation as $\sqrt{N t^{-1}}$. Therefore, the simulation should be carried out long enough such that any systematic error is reduced to a level having negligible influence on the coupled simulation.

Two additional issues must be addressed in the model generation. First, the width of the Surface MD Region will generally not fully contain the domain of atoms having equilibrium states disrupted due to free surface effects. Therefore, an additional simulation will be required to obtain the forces, $\left.\vec{f}_{s}\right|_{\vec{\delta}_{I}=\{0\}}$, in the reference state as explained in Appendix B (Equation (B15)). Second, the elastic anisotropy of the FEM domain is a function of the crystallographic orientation of the atomistic microstructure 
and should be derived from the interatomic potential used in the MD system under the equivalent thermal and mechanical loading conditions to avoid mismatch of elastic properties at the interface.

The operations discussed in this appendix complete the model construction. This model, together with applied far field boundary conditions, is used to start the first MD FEM iteration of the coupled simulation.

A summary of the individual steps involved in developing the complete ESCM model is presented in Figure A2.

\section{APPENDIX B}

\section{Calculation of Compensating Forces}

As discussed in Section 2.3, there are two spurious forces created within the MD system in the ESCM approach that have to be eliminated. One is the surface tension force, $\vec{\tau}$, created by the applied free surface boundary conditions at the perimeter of the MD system. The other is the elastic reaction force, $\vec{\xi}$, of the Surface MD Region created by its stiffness as the MD system deforms. The method to neutralize both of these forces is based on applying an external counterforce to the atoms within the Surface MD Region

$$
\vec{f}_{c}=-(\vec{\xi}+\vec{\tau}) \text {. }
$$

The counterforce is specifically determined for each SVC and is uniformly distributed over the atoms of the SVC so that the total sum of forces in the SVC is zero

$$
\vec{f}_{c}+\vec{\xi}+\vec{\tau}=0
$$

Because $\vec{\xi}$ and $\vec{\tau}$ cannot be estimated directly during the simulation, the expression of the counterforce given in (Equation (B1)) cannot be used to directly determine $\vec{f}_{c}$. What can be determined in the MD simulation, however, is the net spurious force, $\vec{f}_{s}$, that is generated in the surface MD Region and imposed on the remainder of the MD system. As illustrated in Figure 3, the free surface force, $\vec{f}_{s}$, for a given SVC is defined as the force between the SVC and the Interface MD Region. It is calculated individually for 
each SVC as a sum over the pair forces between atoms (i) of the SVC and all of their interaction neighbors $(j)$ lying outside the Surface MD Region

$$
\vec{f}_{s}(t)=\sum_{i \in(S V C)} \sum_{j \notin(\text { surf.layer })} \vec{f}^{(i, j)}(t)
$$

The pair force between atoms $(i)$ and $(j)$ for a potential based on the embedded atom method (EAM) [39] can be expressed as

$$
\vec{f}^{(i, j)}(t)=-\left[\frac{\partial \phi^{(i)}(t)}{\partial r^{(i, j)}}+\frac{\partial \phi^{(j)}(t)}{\partial r^{(i, j)}}\right] \frac{\vec{r}^{(i, j)}}{r^{(i, j)}}
$$

where $\phi^{(i)}(t)$ is the potential energy of atom (i) at time $t$, and $\vec{r}^{(i, j)}=\vec{r}^{(i)}-\vec{r}^{(j)}$ with $r^{(i, j)}=\left|\vec{r}^{(i, j)}\right|$.

To ensure that the equilibrium condition for a perfect crystal lattice is satisfied at zero pressure and $T=0 \mathrm{~K}$, the following condition must be met

$$
\left.\vec{f}_{s}(0)\right|_{P=0, T=0}=\{0\}
$$

A requirement on the division of the Surface MD Region is that each SVC must occupy a whole number of lattice unit cells. The reason for this requirement is because any resultant force at the atomic scale is a sum of attractive and repulsive forces between interacting atoms. The equilibrium condition is satisfied only for the special case of a complete, periodic, lattice unit cell. Conversely, equilibrium is not satisfied for individual atoms or for arbitrarily defined groups of atoms.

During simulation with evolving deformations and with the presence of a free surface, $\vec{f}_{s}$ is equal to (after averaging the thermal fluctuations, inherent in each atomistically calculated force) the sum of $\vec{\xi}$ and that part of $\vec{\tau}$, indicated as $\vec{\tau}_{s}$, which is contained in the Surface MD Region only

$$
\vec{f}_{s}=\vec{\xi}+\vec{\tau}_{s}
$$

In the above equation, an assumption is made that a very thin Surface MD Region of a few nanometers thickness may not contain all the surface tension effects, so that the total surface tension force is decomposed into two components

$$
\vec{\tau}=\vec{\tau}_{s}+\vec{\tau}_{I}
$$


where $\vec{\tau}_{s}$ is the component that is contained in the Surface MD Region, and $\vec{\tau}_{I}$ is the component that is contained in the remaining inner part of the MD system. Only $\vec{\tau}_{s}$ would give contribution to $\vec{f}_{s}$ in Equation (B6).

When the counterforce, $\vec{f}_{c}$, is applied, $\vec{f}_{s}$ now equals

$$
\vec{f}_{s}=\vec{\xi}+\vec{\tau}_{s}+\vec{f}_{c}
$$

Using the definition for $\vec{f}_{c}$ expressed by Equation (B1) for full compensation yields

$$
\vec{f}_{s}=\vec{\xi}+\vec{\tau}_{s}-(\vec{\xi}+\vec{\tau})=-\vec{\tau}_{I}
$$

Equation (B9) gives the criteria for full compensation of the spurious forces as $\vec{f}_{s}=-\vec{\tau}_{I}$. The counterforce, $\vec{f}_{c}$, can now be defined as the force, which is needed to maintain $\vec{f}_{s}(t)=-\vec{\tau}_{I}$. This definition has the desirable feature of not requiring that $\vec{\xi}$ and $\vec{\tau}$ be determined explicitly. An iterative procedure is used to maintain $\vec{f}_{s}(t)=-\vec{\tau}_{I}$ by correcting $\vec{f}_{c}(t)$ within each SVC by the negative of the difference between $\vec{f}_{s}(t)$ and $-\vec{\tau}_{I}$ at any given time $t$, as

$$
\vec{f}_{c}(t+\Delta t)=\vec{f}_{c}(t)-\frac{\Delta t}{t_{M}}\left[\vec{f}_{s}(t)+\vec{\tau}_{I}\right] \quad \vec{f}_{c}(0)=\{0\}
$$

Here, $\Delta t$ is the MD time step, and $t_{\mathrm{M}}>\Delta t$ is an adjustable inertial time parameter that controls the sensitivity of the counterforce to fast atomic fluctuations of the surface force (a suitable choice was found to be $t_{\mathrm{M}}=1000 \Delta t$ ).

In order to perform the iteration in Equation (B10), the surface force component, $\vec{\tau}_{I}$, has to be determined. If the Surface MD Region is thick enough, $\vec{\tau}_{I}$ is a small fraction of $\vec{\tau}$, and a good simplifying assumption is to set $\vec{\tau}_{I}=0$, which reduces Equation (B10) to

$$
\vec{f}_{c}(t+\Delta t)=\vec{f}_{c}(t)-\frac{\Delta t}{t_{M}} \vec{f}_{s}(t) ; \quad \vec{f}_{c}(0)=\{0\}
$$

Otherwise, when $\vec{\tau}_{I}$ is considered non-negligible, the following method can be used for its estimation and is based on two considerations. First, when the MD system is not deformed, the elastic reaction force of the surface region is zero, or $\vec{\xi}=0$. Second, the assumption is made that the deformation does not change the surface tension, which is 
appropriate if changes in the surface curvature and the surface energy due to deformation are negligible.

A non-deformed state is defined when the estimated displacements, $\vec{\delta}_{I}$, are zero. Here, it is recalled that $\vec{\delta}_{I}$ was defined in Equation (1) relative to $\vec{r}_{C M}(0)$ for a defect free system equilibrated under fully $3 \mathrm{D}$ periodic boundary conditions with no free surface. To make $\vec{\delta}_{I}=0$, an external radial force per unit area given by

$$
\vec{f}_{r}=-\vec{\tau}=-\frac{\gamma}{r}
$$

is uniformly applied to the atoms of the surface region having a free surface with surface tension, $\gamma$, and radius of curvature, $r$. Equation (B12) is the Young-Laplace's equation for the internal pressure of a cylindrical particle of radius $r$ due to its surface tension. Even though the Young-Laplace's equation is strictly applicable to liquids, it has been shown that it also provides a reasonable approximation for very small metallic domains [30].

Under the conditions that $\vec{\delta}_{I}=0$ and $\vec{\xi}=0$, only $\vec{\tau}_{s}$ and $\vec{f}_{r}$ would contribute to $\vec{f}_{s}$, which can be presented as

$$
\vec{f}_{s}=\vec{\tau}_{s}+\vec{f}_{r}=\vec{\tau}_{s}-\vec{\tau}=-\vec{\tau}_{I}
$$

Expressed in another way, recalling Equation (B3), $\vec{\tau}_{I}$ is defined as:

$$
\vec{\tau}_{I}=-\left.\vec{f}_{s}\right|_{\vec{\delta}_{I}=\{0\}}=-\sum_{i \in(\text { SVC }) j \notin(\text { surf.layer })} \vec{f}_{\vec{\delta}_{I}=\{0\}}
$$

Equation (B14) allows $\vec{\tau}_{I}$ to be calculated through the atomistic forces in an equilibrated MD system when $\vec{\delta}_{I}=0$. To avoid uncertainties from random thermal fluctuations in the atomistic calculation of $\left.\vec{f}_{s}\right|_{\vec{\delta}_{I}=\{0\}}$, a suitable time averaging at equilibrium can be used in Equation (B14).

After substituting Equation (B14) in Equation (B10), the iteration procedure for calculating the counterforce becomes

$$
\vec{f}_{c}(t+\Delta t)=\vec{f}_{c}(t)-\frac{\Delta t}{t_{M}}\left[\vec{f}_{s}(t)-\left.\vec{f}_{s}\right|_{\vec{\delta}_{I}=\{0\}}\right] \quad \vec{f}_{c}(0)=\{0\}
$$

The iteration in Equation (B15) is performed separately for each SVC to obtain and update $\vec{f}_{c}(t)$ at every MD time step to counteract both $\vec{\xi}$ and $\vec{\tau}$ at the same time during 
the coupled simulation by maintaining $\left.\vec{f}_{s}(t) \approx \vec{f}_{s}\right|_{\vec{\delta}_{I}=\{0\}}$. Introducing the initial force state $\left.\vec{f}_{s}\right|_{\vec{\delta}_{I}=\{0\}}$ as a reference state in Equation (B15) allows the use of a very thin Surface MD Region (1 to $2 \mathrm{~nm}$ has been used in the present study), so that its stiffness could be small and the compensating force, $\vec{f}_{c}(t)$, would be a small perturbation to the interatomic forces. For a thicker Surface MD Region, $\vec{\tau}_{I} \rightarrow 0$, and the simplified iteration (Equation (B11)) can be used instead. 
FIGURES

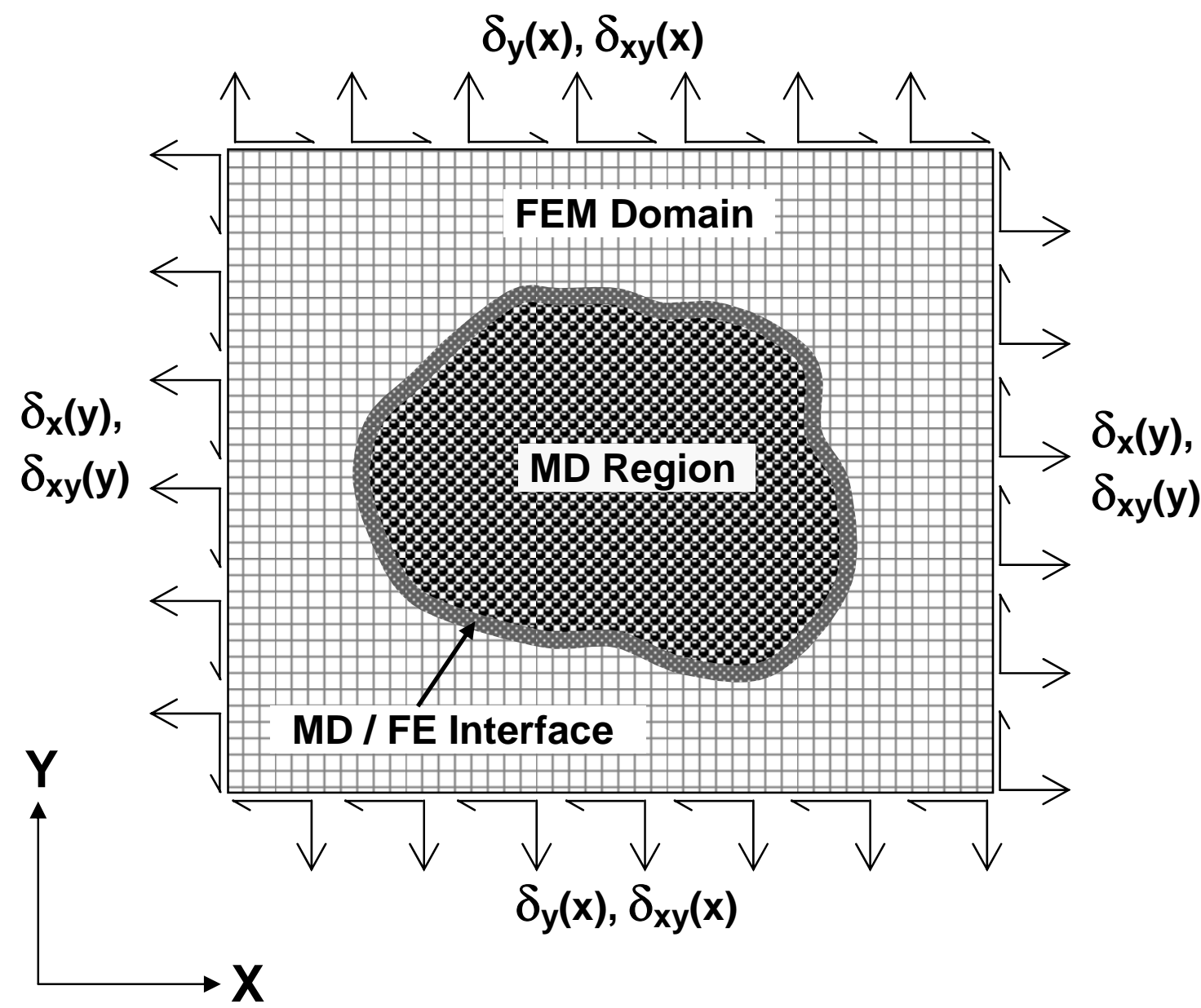

Figure 1. Model topology used in the ESCM approach depicting the continuum region represented by the finite element method (FEM), the atomistic domain simulated by molecular dynamics (MD), and the $\mathrm{MD} / \mathrm{FE}$ Interface region coupling the two computational approaches. 
To be submitted to International Journal for Numerical Methods in Engineering

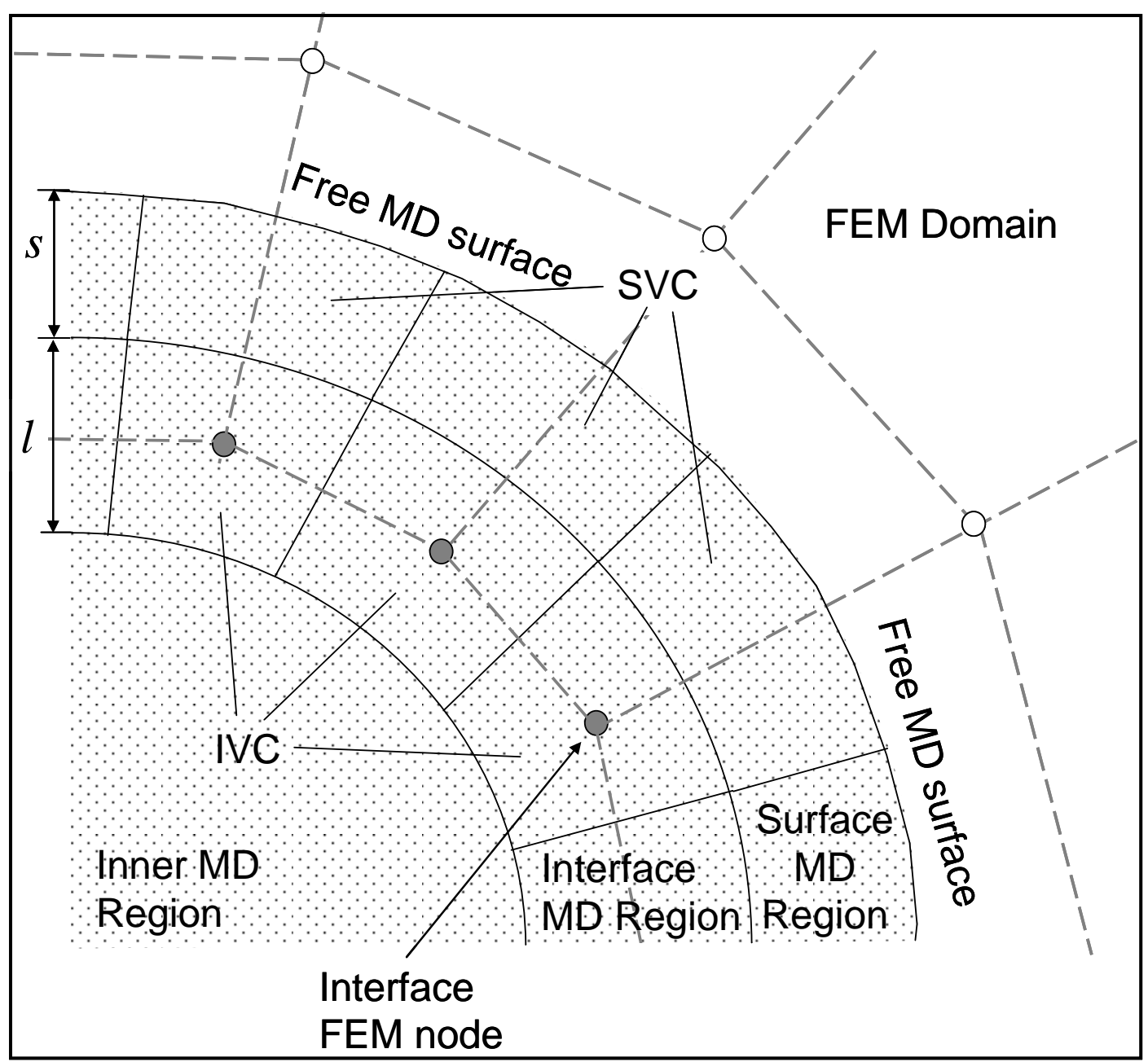

Figure 2. Structure of the MD/FE Interface in the ESCM approach. 


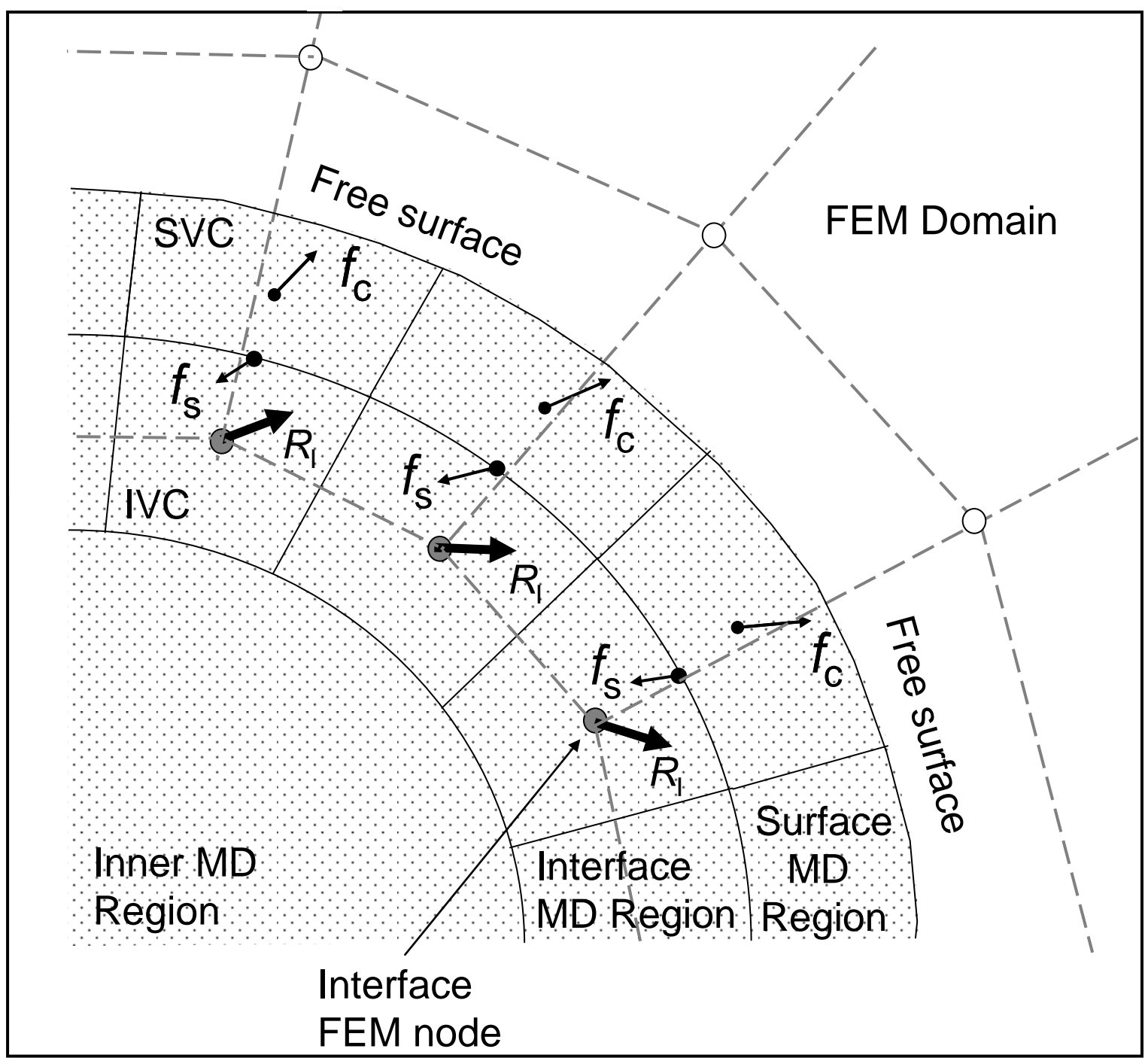

Figure 3. Forces within the Interface and Surface MD Regions. $R_{I}$ are FEM reaction forces, $f_{s}$ are the resultant surface region forces, and $f_{c}$ are applied compensating forces. 


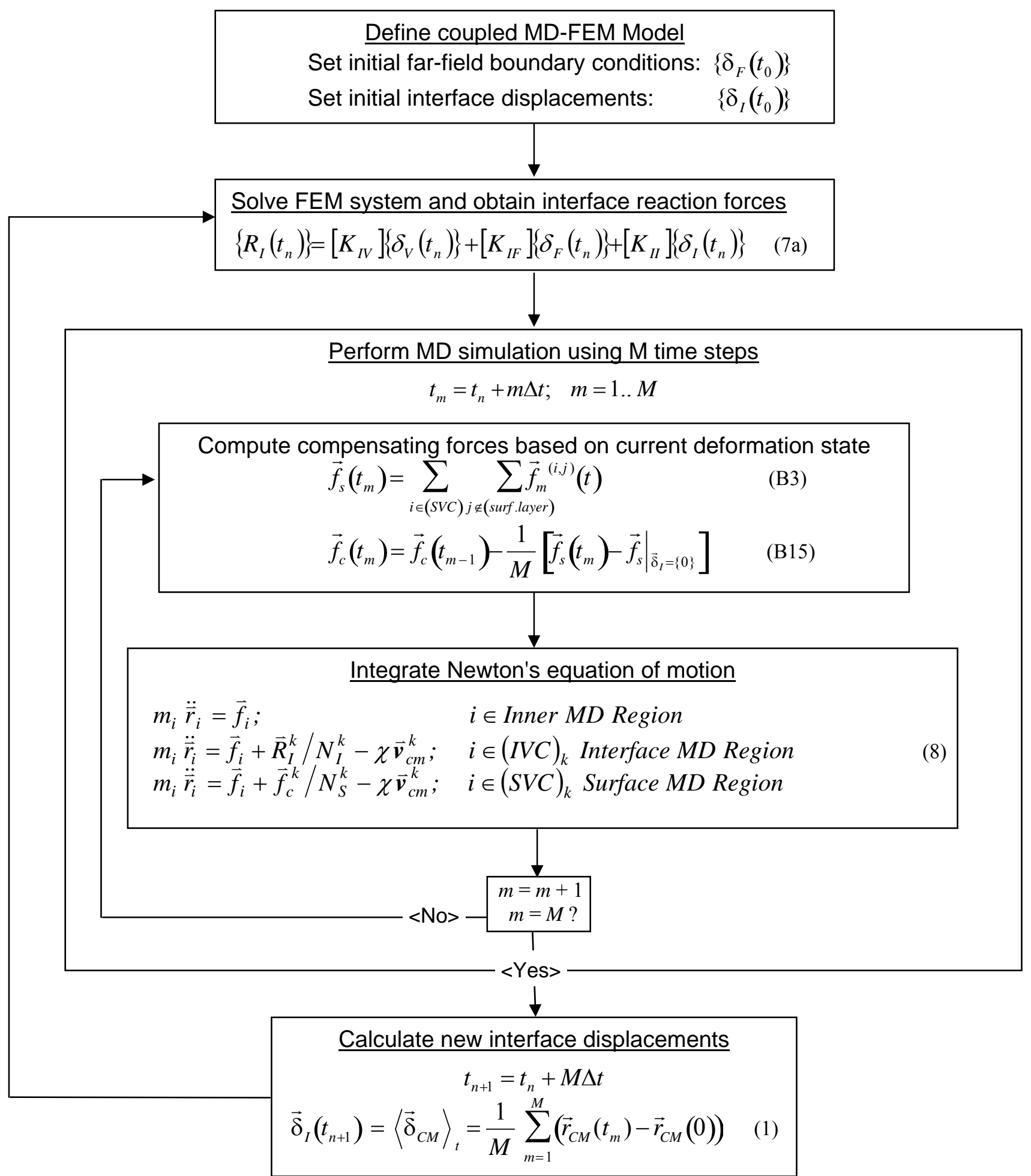

Figure 4. Flowchart summarizing the coupled analysis procedure. 
To be submitted to International Journal for Numerical Methods in Engineering

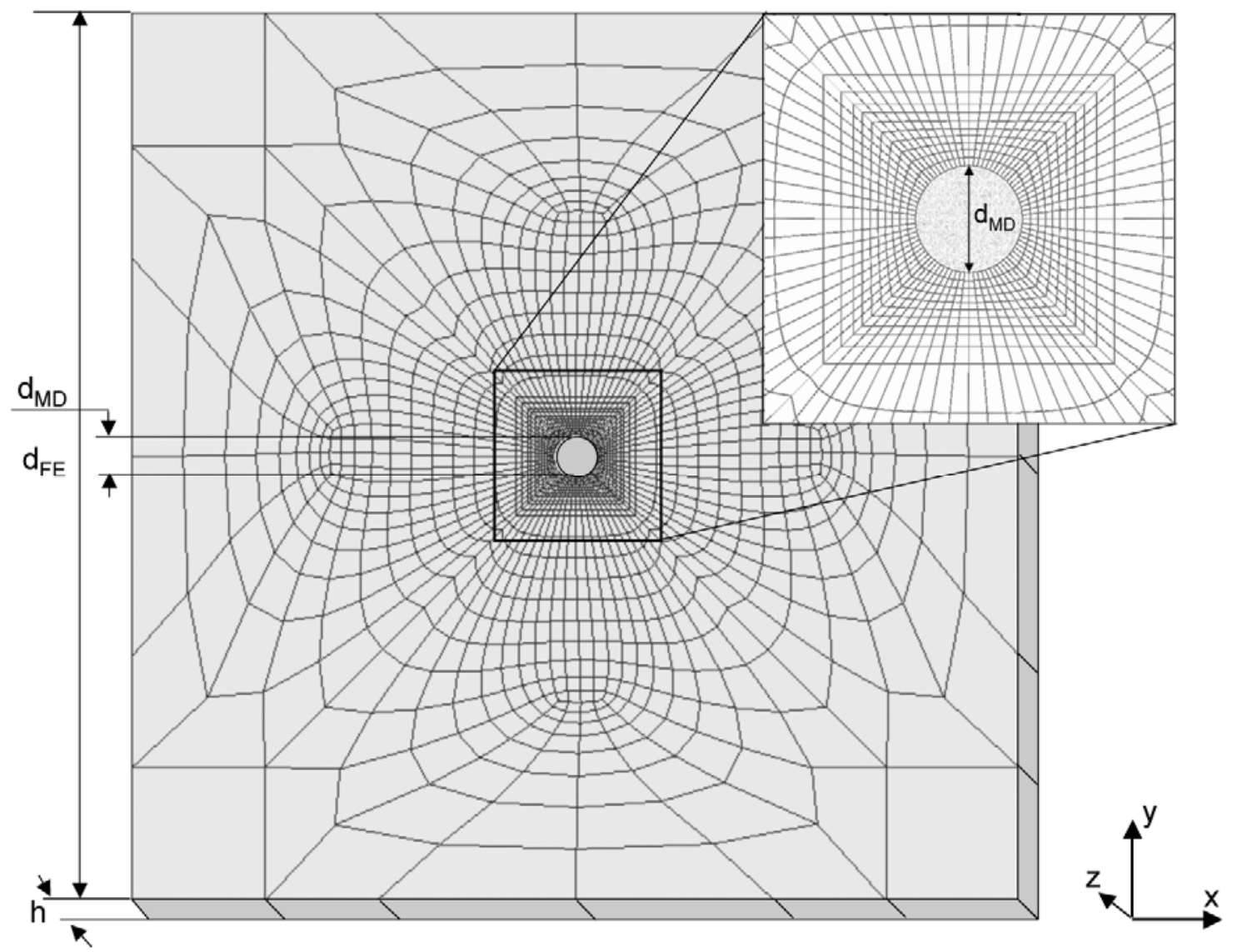

Figure 5. Model geometry of the test MD-FEM coupled system. 


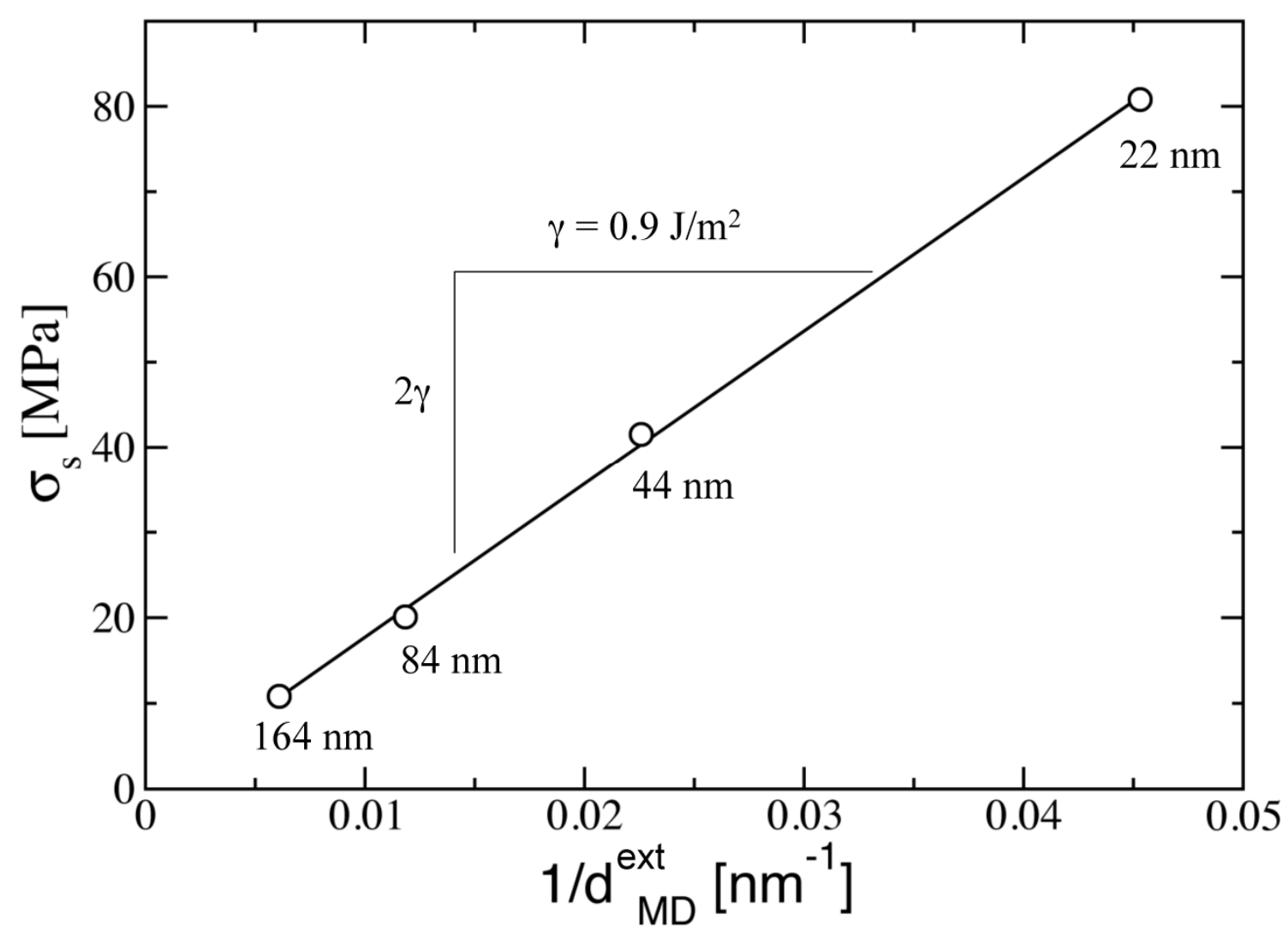

Figure 6. Dependence of the pressure $p_{\mathrm{s}}$ caused by the surface tension of a circular MD domain of radius $d_{M D}$ simulated with free surface boundary conditions. The slope is proportional to the surface tension $\gamma$. 


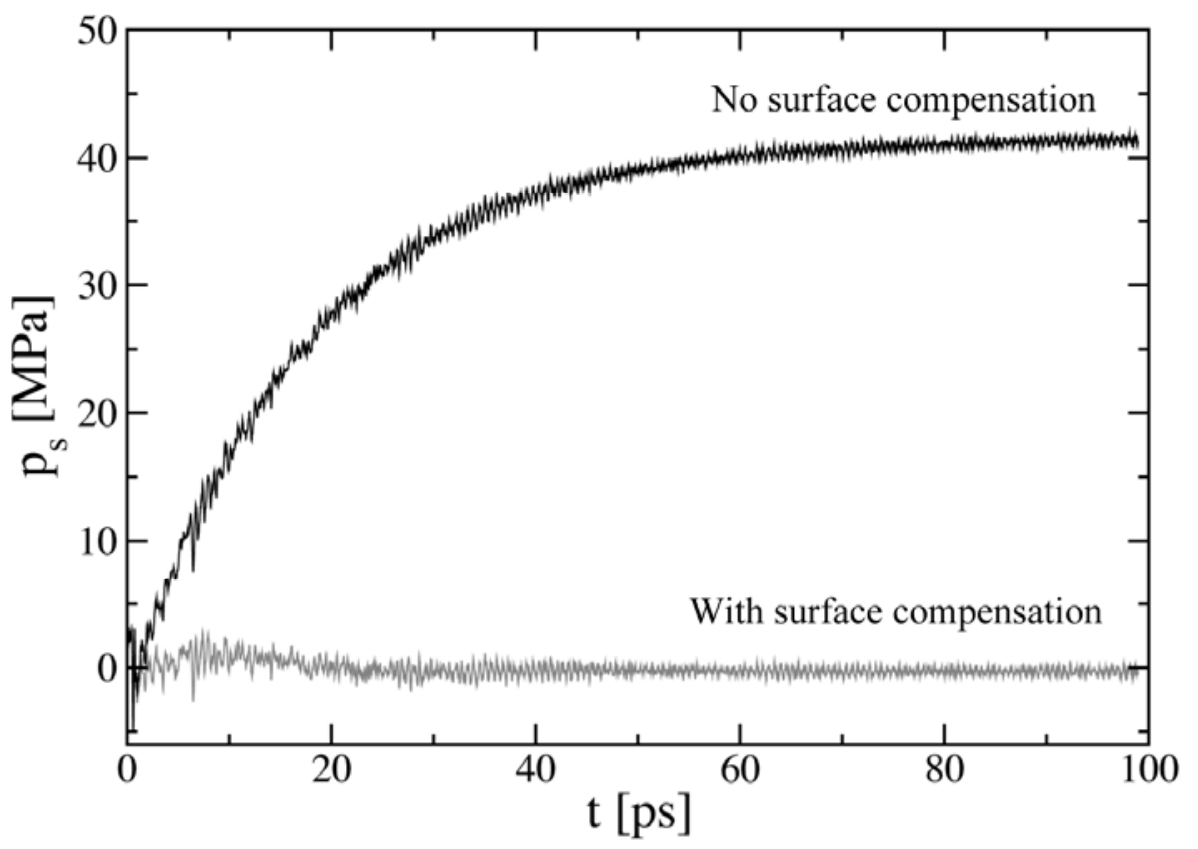

Figure 7. Evolution of the internal pressure of a circular MD system of $d_{M D}=44 \mathrm{~nm}$ with and without surface tension compensation. 


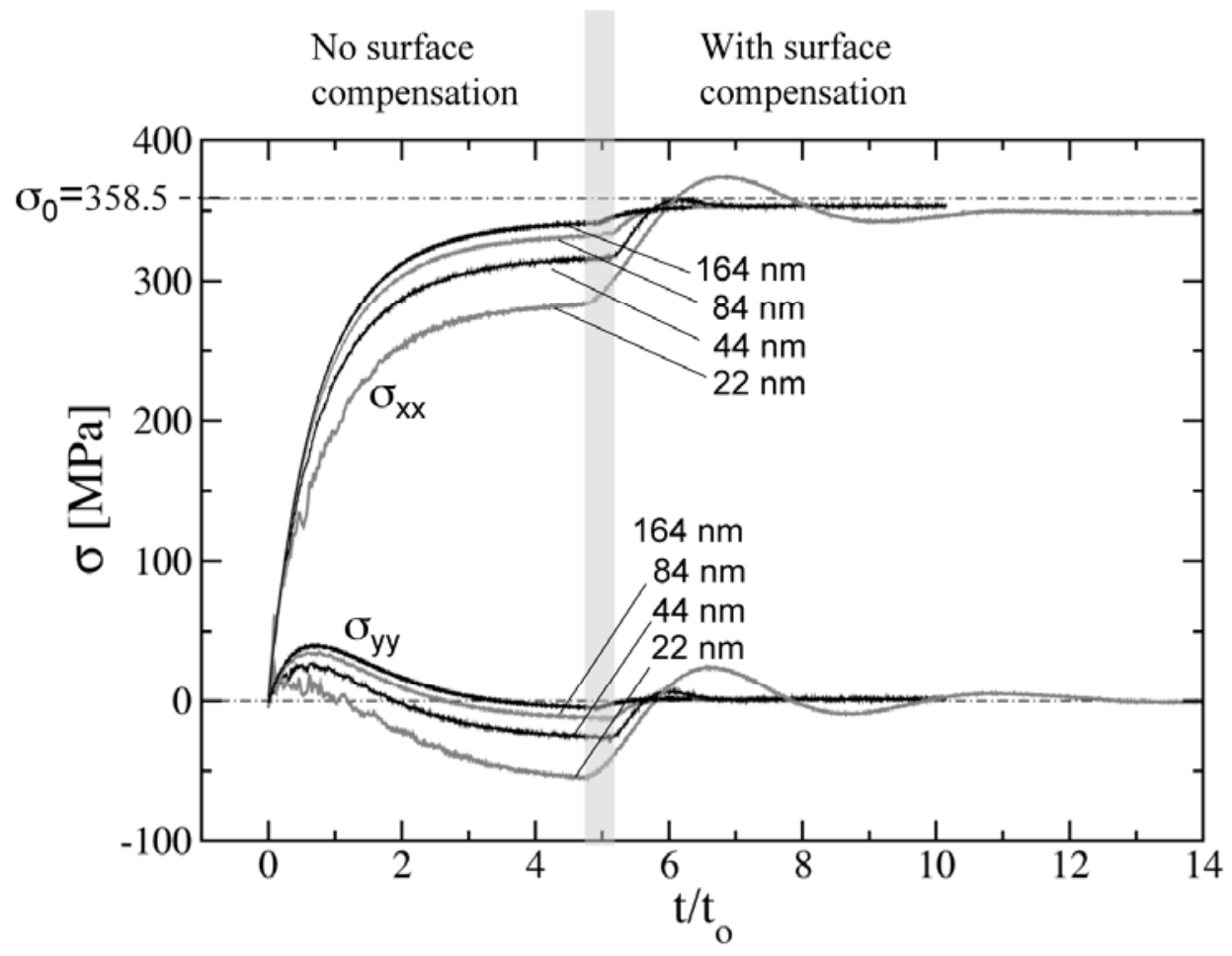

Figure 8. Evolution of the longitudinal tensile $\sigma_{\mathrm{xx}}$ and transverse $\sigma_{\mathrm{yy}}$ stress components of the internal stress of the MD system of a coupled MD-FE model simulated at $0.5 \%$ far field strain. Data for four circular MD systems of diameters from 22 to $164 \mathrm{~nm}$ is given before $\left(t / t_{0}<5\right)$ and after $\left(t / t_{0}>5\right)$ Surface MD Region compensation (shown separated by the gray band). 


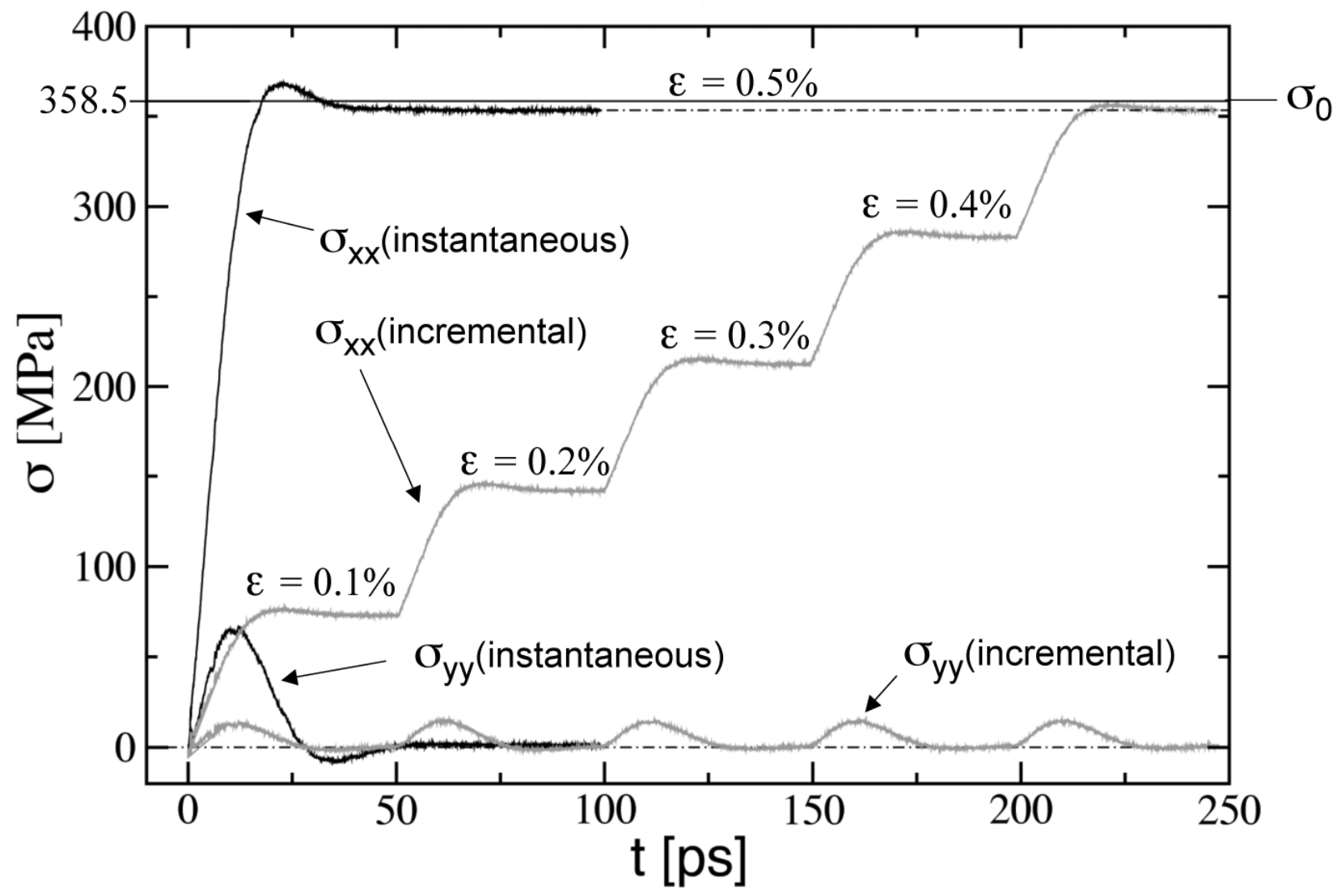

Figure 9. Dynamic response of the coupled MD-FEM system under far-field homogeneous tensile strain $\varepsilon_{\mathrm{xx}}$ applied either instantaneously (black) or in five consecutive increments (gray). 


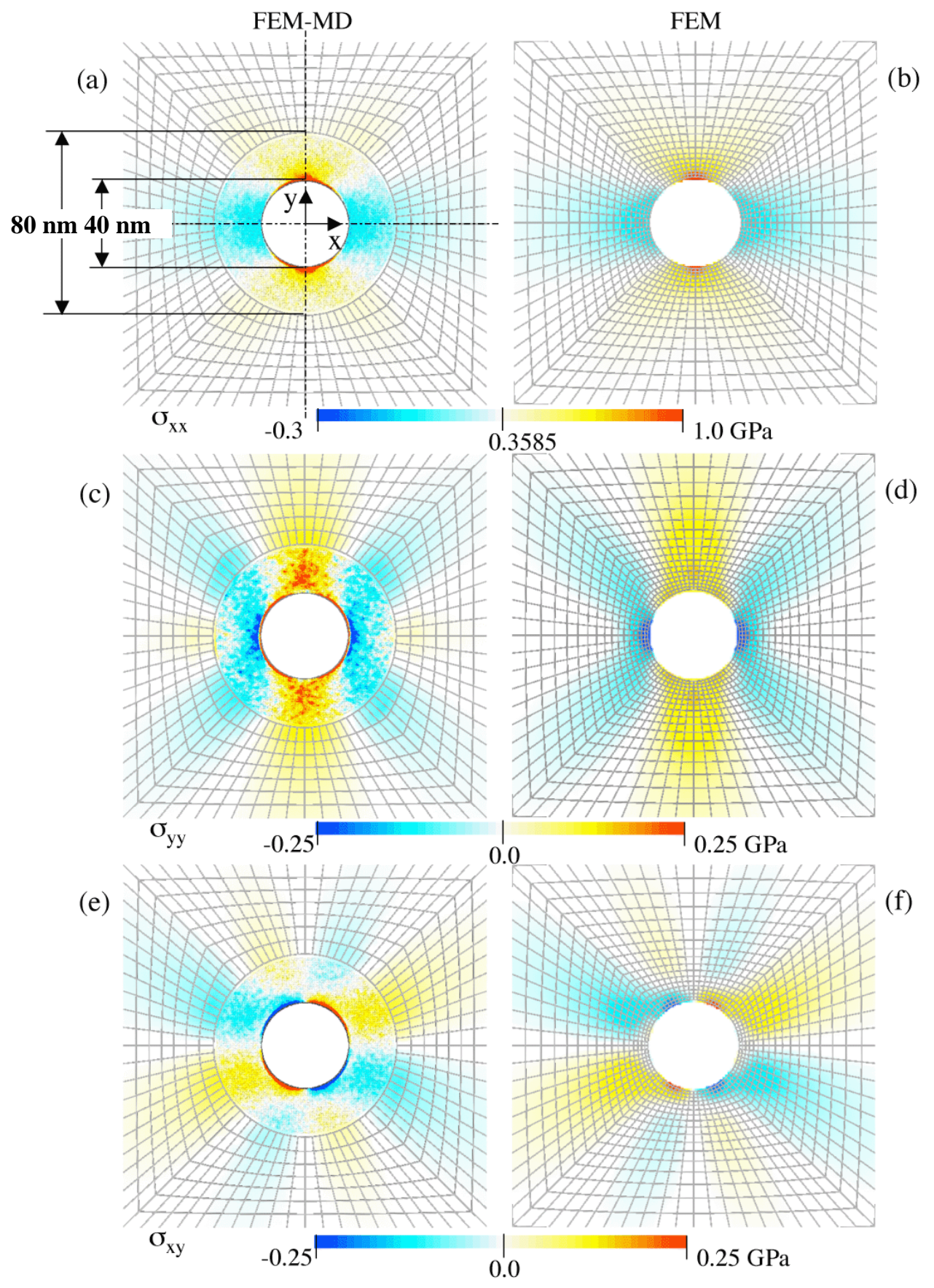

Figure 10 Simulation of an open hole specimen. Comparison of the stress field for $\sigma_{\mathrm{xx}}, \sigma_{\mathrm{yy}}$, and $\sigma_{x y}$ stress components of the coupled MD-FEM system (a, c, e) with a full threedimensional anisotropic continuum FEM solution (b, d, f). 

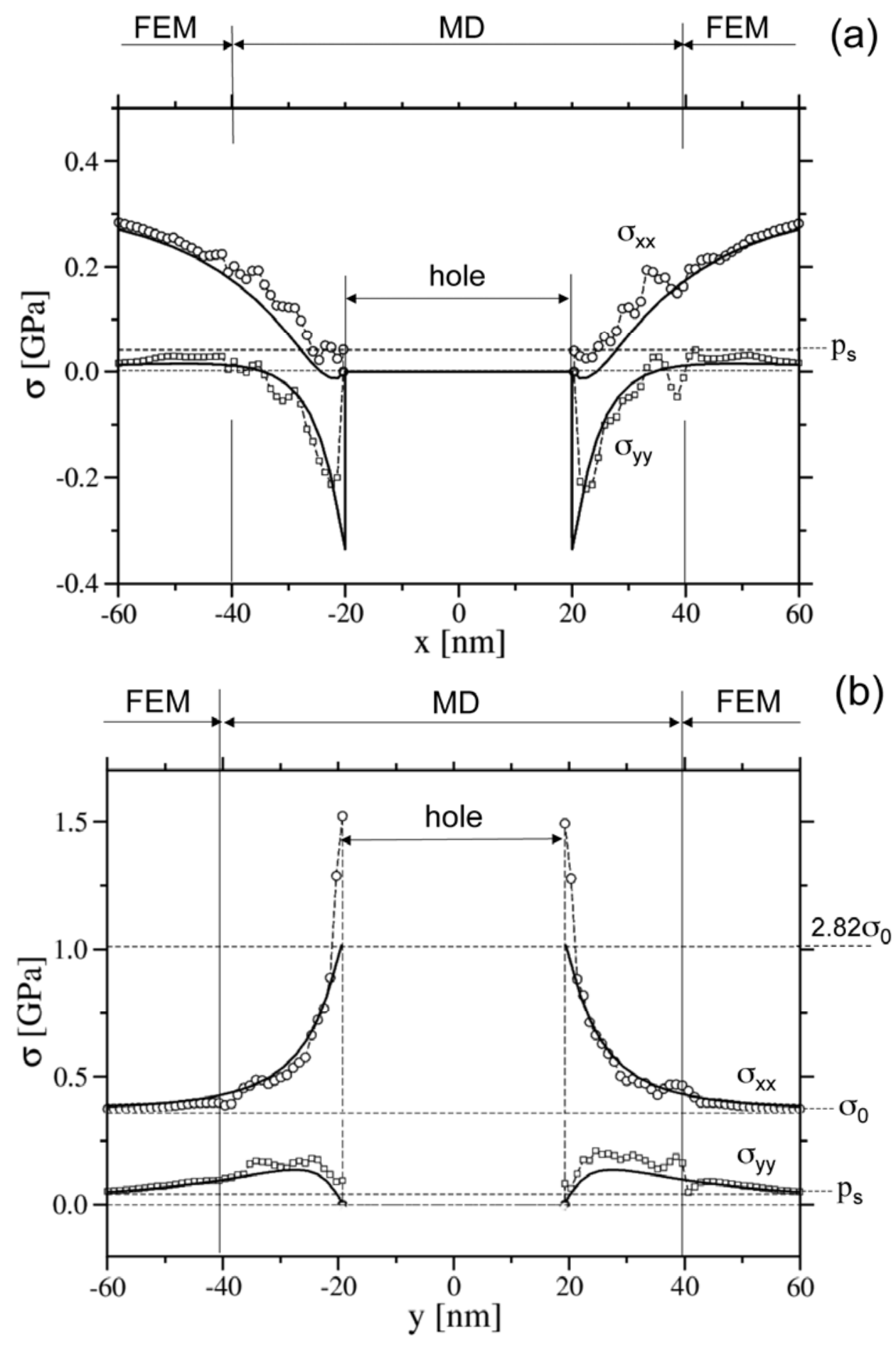

Figure 11 Stress profiles in the open hole specimen for $\sigma_{x x}$ and $\sigma_{y y}$ along (a) the $x$-axis and (b) the y-axis scanned through the center of the hole (as given in Figure 11(a)). The symbols represent the ESCM simulation data, while the full lines represent fully continuum FEM results. For reference, the surface tension induced pressure $p_{\mathrm{s}}$ is also shown. 


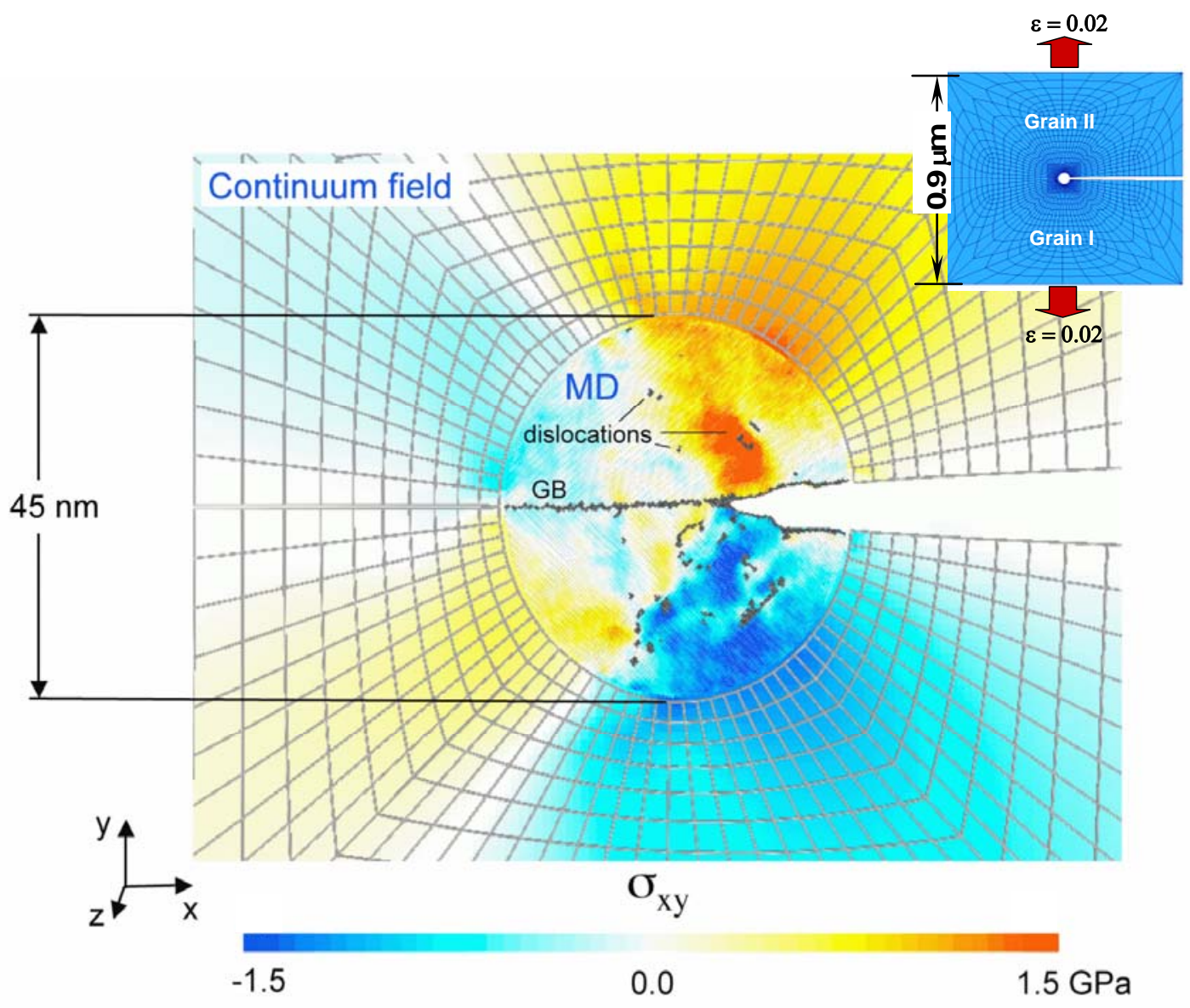

Figure 12 ESCM simulation of an edge crack propagating along a $<110>/ \Sigma 99$ symmetric tilt grain boundary in $\mathrm{Al}$ at $2 \%$ far-field uniaxial strain showing shear stress $\sigma_{\mathrm{xy}}$. 


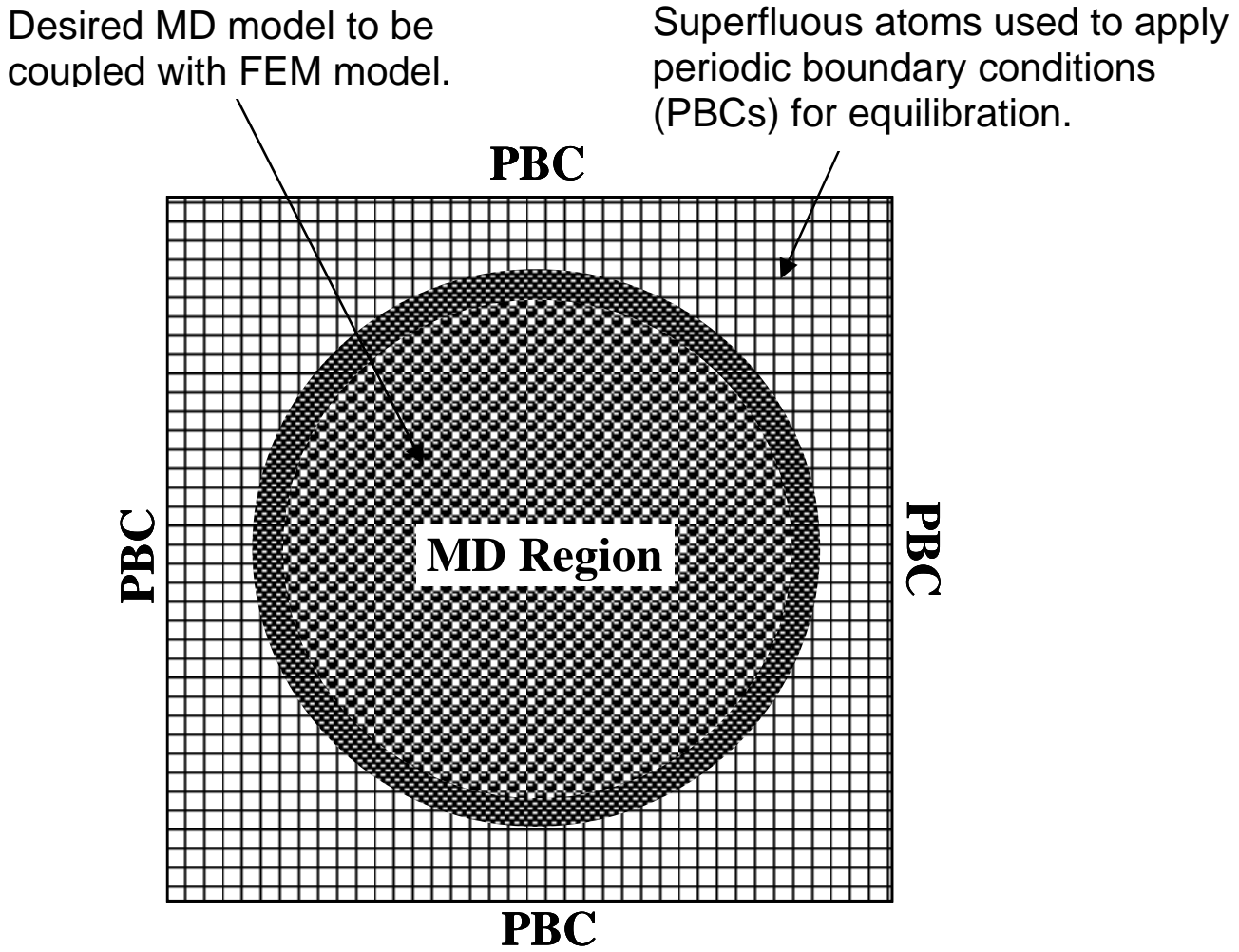

Figure A1. Generation of MD model by equilibrating within a larger rectangular MD model under PBCs. 


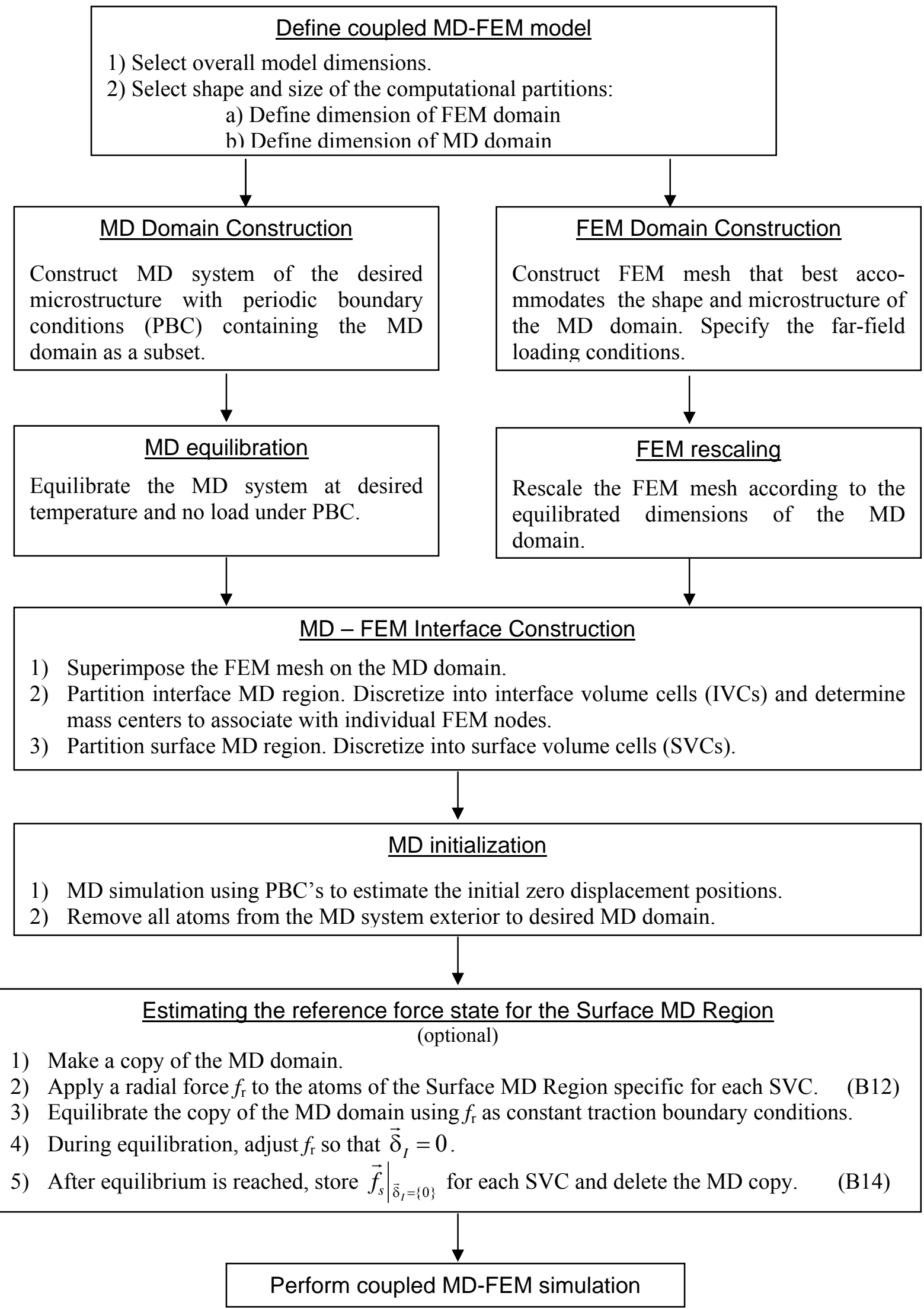

Figure A2. Flowchart summarizing the MD-FE model construction. 